ESAIM: M2AN 49 (2015) 481-502

DOI: $10.1051 / \mathrm{m} 2 \mathrm{an} / 2014041$
ESAIM: Mathematical Modelling and Numerical Analysis

www.esaim-m2an.org

\title{
A NITSCHE FINITE ELEMENT METHOD FOR DYNAMIC CONTACT: 1. SPACE SEMI-DISCRETIZATION AND TIME-MARCHING SCHEMES
}

\author{
Franz Chouly ${ }^{1}$, Patrick Hild ${ }^{2}$ and Yves Renard ${ }^{3}$
}

\begin{abstract}
This paper presents a new approximation of elastodynamic frictionless contact problems based both on the finite element method and on an adaptation of Nitsche's method which was initially designed for Dirichlet's condition. A main interesting characteristic is that this approximation produces well-posed space semi-discretizations contrary to standard finite element discretizations. This paper is then mainly devoted to present an analysis of the space semi-discretization in terms of consistency, well-posedness and energy conservation, and also to study the well-posedness of some time-marching schemes ( $\theta$-scheme, Newmark and a new hybrid scheme). The stability properties of the schemes and the corresponding numerical experiments can be found in a second paper [F. Chouly, P. Hild and Y. Renard, A Nitsche finite element method for dynamic contact. 2. Stability analysis and numerical experiments. ESAIM: M2AN 49 (2015) 503-528.].
\end{abstract}

Mathematics Subject Classification. 65N12, 65N30, 74M15.

Received march 13, 2014. Revised September 1st, 2014.

Published online 17 March 2015.

\section{INTRODUCTION AND PROBLEM SETTING}

The numerical implementation of contact and impact problems in solid mechanics generally uses the Finite Element Method (FEM) (see $[23,25,29,35,37,46,47])$. In this paper we propose an extension to the elastodynamics framework of the Nitsche-based method introduced previously in $[13,14]$ in the case of unilateral contact in elastostatics. Although we restrict ourselves to the unilateral contact without friction in this study, it should be noted that Nitsche's method can be extended without much difficulty to the case of frictional unilateral contact (see $[12,43])$.

Nitsche's method [40] aims at treating the boundary or interface conditions in a weak sense, thanks to a consistent penalty term. So it differs from standard penalization techniques which are typically non-consistent [35]. Moreover, unlike mixed methods (see, e.g., $[29,46]$ ), no additional unknown (Lagrange multiplier) is needed. Nitsche's method has been widely applied during these last years to problems involving linear conditions on the

\footnotetext{
Keywords and phrases. Unilateral contact, elastodynamics, finite elements, Nitsche's method, time-marching schemes, stability.

1 Laboratoire de Mathématiques de Besançon - UMR CNRS 6623, Université de Franche Comté, 16 route de Gray, 25030 Besançon cedex, France. franz.chouly@univ-fcomte.fr

2 Institut de Mathématiques de Toulouse - UMR CNRS 5219, Université Paul Sabatier, 118 route de Narbonne, 31062 Toulouse cedex 9, France. patrick.hild@math.univ-toulouse.fr

3 Université de Lyon, CNRS, INSA-Lyon, ICJ UMR5208, LaMCoS UMR5259, 69621 Villeurbanne, France.

yves.renard@insa-lyon.fr
} 
boundary of a domain or in the interface between sub-domains: see, e.g., [44,45] for the Dirichlet problem, [7] for domain decomposition with non-matching meshes and [27] for a global review. More recently, in [26,32] it has been adapted for bilateral (persistent) contact, which still involves linear boundary conditions on the contact zone. An extension to large strain bilateral contact has been performed in [48].

Concerning time-evolution equations we can make the same observation that only the case of linear boundary/interface conditions has been dealt with. Some works treat in particular the problem of Dirichlet boundary conditions for parabolic equations $[11,31,45]$. Some other works are focused on interface conditions, in the context of transient fluid-structure interaction [5,10,28] or transient Stokes Darcy coupling [18].

The paper is outlined as follows: in Section 2, we first adapt Nitsche's concept to the space semi-discretized problem which is shown to be consistent contrary to the penalty approach. We also show that, when applied to contact-impact in elastodynamics, Nitsche's method has the good property of leading to a well-posed space (semi-)discretization (system of Lipschitz differential equations), which is not the case of standard FEM discretization which leads to an ill-posed measure differential inclusion (by "standard FEM" we mean a mixed method with a Lagrange multiplier that stands for the contact stress, see, e.g., [34], see also Remark 2.2 for further explanations). Note that this well-posedness characteristic of Nitsche's method is also shared by the penalty method and modified mass methods (see [34,42]). We then prove that the symmetric variant of Nitsche's space semi-discretization also conserves an augmented energy, as the penalty method. In Section 3, we define some classical schemes ( $\theta$-scheme, Newmark) in the Nitsche context as well as a new hybrid scheme which satisfies among others some interesting stability properties (see [15]). For any scheme, we then obtain the corresponding CFL conditions ensuring well-posedness.

A second part [15] concerns the theoretical study of stability and energy conservation properties of the aforementioned fully discrete schemes. For the $\theta$-scheme and the Newmark scheme, combined to the symmetric variant of Nitsche, the unconditional stability can be guaranteed only for the most dissipative schemes. Conversely, for the new hybrid scheme, there is no such price to pay for unconditional stability, though small numerical dissipation occurs during the contact phase. The second part contains also numerical experiments, to illustrate stability and energy conservation properties, and additionally to study the presence of spurious oscillations on the solution.

Let us introduce some useful notations. In what follows, bold letters like $\mathbf{u}, \mathbf{v}$, indicate vector or tensor valued quantities, while the capital ones (e.g., V, K ...) represent functional sets involving vector fields. As usual, we denote by $\left(H^{s}(.)\right)^{d}, s \in \mathbb{R}, d=1,2,3$ the Sobolev spaces in one, two or three space dimensions (see [1]). The usual scalar product of $\left(H^{s}(D)\right)^{d}$ is denoted by $(\cdot, \cdot)_{s, D}$, and $\|\cdot\|_{s, D}=(\cdot, \cdot)_{s, D}^{\frac{1}{2}}$ denotes the corresponding norm. We keep the same notation when $d=1$ or $d>1$. We note $\mathscr{C}^{0}(I ; V)$ the space of continuous functions that map $I \subset \mathbb{R}$ into a normed vectorial space $V$. The letter $C$ stands for a generic constant, independent of the discretization parameters.

We consider an elastic body $\Omega$ in $\mathbb{R}^{d}$ with $d=1,2,3$. Small strain assumptions are made (as well as plane strain when $d=2)$. The boundary $\partial \Omega$ of $\Omega$ is polygonal $(d=2)$ or polyhedral $(d=3)$. The outward unit normal vector on $\partial \Omega$ is denoted $\mathbf{n}$. We suppose that $\partial \Omega$ consists in three nonoverlapping parts $\Gamma_{D}, \Gamma_{N}$ and the contact boundary $\Gamma_{C}$, with meas $\left(\Gamma_{D}\right)>0$ and meas $\left(\Gamma_{C}\right)>0$. The contact boundary is supposed to be a straight line segment when $d=2$ or a polygon when $d=3$ to simplify. In the reference configuration, the body is in contact on $\Gamma_{C}$ with a rigid foundation and we suppose that the unknown contact zone during deformation is included into $\Gamma_{C}$. The body is clamped on $\Gamma_{D}$ for the sake of simplicity. It is subjected to volume forces $\mathbf{f}$ in $\Omega$ and to surface loads $\mathbf{g}$ on $\Gamma_{N}$.

We consider the unilateral contact problem in linear elastodynamics during a time interval $[0, T)$, where $T>0$ is the final time. We denote by $\Omega_{T}:=(0, T) \times \Omega$ the time-space domain, and similarly $\Gamma_{D T}:=(0, T) \times \Gamma_{D}$, $\Gamma_{N T}:=(0, T) \times \Gamma_{N}$ and $\Gamma_{C T}:=(0, T) \times \Gamma_{C}$. The problem then consists in finding the displacement field 
$\mathbf{u}:[0, T) \times \Omega \rightarrow \mathbb{R}^{d}$ verifying the equations and conditions (1.1) and (1.2):

$$
\begin{array}{rlrl}
\rho \ddot{\mathbf{u}}-\operatorname{div} \boldsymbol{\sigma}(\mathbf{u}) & =\mathbf{f} & & \text { in } \Omega_{T}, \\
\boldsymbol{\sigma}(\mathbf{u}) & =\mathbf{A} \boldsymbol{\varepsilon}(\mathbf{u}) & & \text { in } \Omega_{T}, \\
\mathbf{u} & =\mathbf{0} & & \text { on } \Gamma_{D T}, \\
\boldsymbol{\sigma}(\mathbf{u}) \mathbf{n} & =\mathbf{g} & & \text { on } \Gamma_{N T}, \\
\mathbf{u}(0, \cdot) & =\mathbf{u}_{0} & \text { in } \Omega, \\
\dot{\mathbf{u}}(0, \cdot) & =\dot{\mathbf{u}}_{0} & & \text { in } \Omega,
\end{array}
$$

where the notation $\dot{\mathbf{x}}$ is used for the time-derivative of a vector field $\mathbf{x}$ on $\Omega_{T}$, so that $\dot{\mathbf{u}}$ is the velocity of the elastic body and $\ddot{\mathbf{u}}$ its acceleration; $\mathbf{u}_{0}$ is the initial displacement and $\dot{\mathbf{u}}_{0}$ is the initial velocity. The density of the elastic material denoted by $\rho$ is supposed to be constant to simplify (this is not restrictive and the results can be extended straightforwardly for a variable density). The notation $\boldsymbol{\sigma}=\left(\sigma_{i j}\right), 1 \leq i, j \leq d$, stands for the stress tensor field and div denotes the divergence operator of tensor valued functions. The notation $\varepsilon(\mathbf{v})=\left(\boldsymbol{\nabla} \mathbf{v}+\boldsymbol{\nabla} \mathbf{v}^{T}\right) / 2$ represents the linearized strain tensor field and $\mathbf{A}$ is the fourth order symmetric elasticity tensor having the usual uniform ellipticity and boundedness property. For any displacement field $\mathbf{v}$ and for any density of surface forces $\boldsymbol{\sigma}(\mathbf{v}) \mathbf{n}$ defined on $\partial \Omega$ we adopt the following notation:

$$
\mathbf{v}=v_{n} \mathbf{n}+\mathbf{v}_{\mathbf{t}} \quad \text { and } \quad \boldsymbol{\sigma}(\mathbf{v}) \mathbf{n}=\sigma_{n}(\mathbf{v}) \mathbf{n}+\boldsymbol{\sigma}_{\mathbf{t}}(\mathbf{v}),
$$

where $\mathbf{v}_{\mathbf{t}}\left(\right.$ resp. $\left.\boldsymbol{\sigma}_{\mathbf{t}}(\mathbf{v})\right)$ are the tangential components of $\mathbf{v}$ (resp. $\left.\boldsymbol{\sigma}(\mathbf{v}) \mathbf{n}\right)$. The conditions describing unilateral contact without friction on $\Gamma_{C T}$ are:

$$
\begin{aligned}
u_{n} & \leq 0 \\
\sigma_{n}(\mathbf{u}) & \leq 0 \\
\sigma_{n}(\mathbf{u}) u_{n} & =0 \\
\boldsymbol{\sigma}_{\mathbf{t}}(\mathbf{u}) & =0
\end{aligned}
$$

Note additionally that the initial displacement $\mathbf{u}_{0}$ should satisfy the compatibility condition $u_{0 n} \leq 0$ on $\Gamma_{C}$.

We introduce the following Hilbert spaces:

$$
\begin{aligned}
\mathbf{V} & :=\left\{\mathbf{v} \in\left(H^{1}(\Omega)\right)^{d}: \mathbf{v}=\mathbf{0} \text { on } \Gamma_{D}\right\} \\
\mathcal{W} & :=\left\{\mathbf{v} \in L^{2}(0, T ; \mathbf{V}): \dot{\mathbf{v}} \in L^{2}\left(0, T ;\left(L^{2}(\Omega)\right)^{d}\right)\right\},
\end{aligned}
$$

which are endowed respectively with the inner products:

$$
\begin{array}{r}
(\mathbf{v}, \mathbf{w})_{\mathbf{V}}:=(\mathbf{v}, \mathbf{w})_{1, \Omega}=\int_{\Omega} \mathbf{v} \cdot \mathbf{w} \mathrm{d} \Omega+\int_{\Omega} \nabla \mathbf{v}: \nabla \mathbf{w} \mathrm{d} \Omega \quad \forall \mathbf{v}, \mathbf{w} \in \mathbf{V}, \\
(\mathbf{v}, \mathbf{w})_{\mathcal{W}}:=\int_{0}^{T}\left(\mathbf{v}(t), \mathbf{w}(t)_{\mathbf{V}} \mathrm{d} t+\int_{0}^{T}\left(\int_{\Omega} \dot{\mathbf{v}}(t) \cdot \dot{\mathbf{w}}(t) \mathrm{d} \Omega\right) \mathrm{d} t \quad \forall \mathbf{v}, \mathbf{w} \in \mathcal{W},\right.
\end{array}
$$

where we used the notation $\mathbf{A}: \mathbf{B}:=\sum_{1 \leq i, j \leq d} A_{i j} B_{i j}$ for the tensors $\mathbf{A}=\left(A_{i j}\right)$ and $\mathbf{B}=\left(B_{i j}\right)$ (about the space $\mathcal{W}$, the interested reader may refer to, e.g., [19]). We define the convex cone $\mathcal{K}$ of admissible displacements which satisfy the noninterpenetration on the contact zone $\Gamma_{C}$ :

$$
\mathcal{K}:=\left\{\mathbf{v} \in \mathcal{W}: v_{n}(t, \cdot) \leq 0 \text { a.e. on } \Gamma_{C} \text { for a.e. } t \in(0, T)\right\} .
$$

Suppose that $\mathbf{u}_{0} \in \mathbf{V}$, with $u_{0 n} \leq 0$ a.e. on $\Gamma_{C}$, and that $\dot{\mathbf{u}}_{0} \in\left(L^{2}(\Omega)\right)^{d}$. Suppose also that $\mathbf{f} \in$ $\mathscr{C}^{0}\left([0, T] ;\left(L^{2}(\Omega)\right)^{d}\right)$ and $\mathbf{g} \in \mathscr{C}^{0}\left([0, T] ;\left(L^{2}\left(\Gamma_{N}\right)\right)^{d}\right)$, which imply that they belong respectively to $\left(L^{2}\left(\Omega_{T}\right)\right)^{d}$ and $\left(L^{2}\left(\Gamma_{N T}\right)\right)^{d}$. 
A weak formulation of Problems (1.1) and (1.2) reads as:

$$
\left\{\begin{array}{l}
\text { Find } \mathbf{u} \in \mathcal{K}, \mathbf{u}(0, \cdot)=\mathbf{u}_{0}, \text { such that : } \\
-\int_{\Omega} \rho \dot{\mathbf{u}}_{0} \cdot\left(\mathbf{v}(0, \cdot)-\mathbf{u}_{0}\right) \mathrm{d} \Omega-\int_{\Omega_{T}} \rho \dot{\mathbf{u}} \cdot(\dot{\mathbf{v}}-\dot{\mathbf{u}}) \mathrm{d} \Omega \mathrm{d} t+\int_{\Omega_{T}} \boldsymbol{\sigma}(\mathbf{u}): \varepsilon(\mathbf{v}-\mathbf{u}) \mathrm{d} \Omega \mathrm{d} t \\
\geq \int_{\Omega_{T}} \mathbf{f} \cdot(\mathbf{v}-\mathbf{u}) \mathrm{d} \Omega \mathrm{d} t+\int_{\Gamma_{N T}} \mathbf{g} \cdot(\mathbf{v}-\mathbf{u}) \mathrm{d} \Gamma \mathrm{d} t \\
\text { for all } \mathbf{v} \in \mathcal{K} \text { for which there exists } \zeta>0 \text { with } \mathbf{v}=\mathbf{u} \text { for } t \geq T-\zeta .
\end{array}\right.
$$

To our knowledge, the well-posedness of Problem (1.3) is still an open issue. The few available existence results concern simplified model problems involving the (scalar) wave equation with Signorini's conditions (see, e.g., $[17,36,39]$ ) or thin structures like membranes, beams (see [2]) or plates (see [41]). Even in these simplified cases, obtention of uniqueness and energy conservation still involves difficulties in 2D or 3D. For a review on some of these results, one can refer to the book [21].

Let us define now the following forms:

$$
a(\mathbf{u}, \mathbf{v}):=\int_{\Omega} \boldsymbol{\sigma}(\mathbf{u}): \varepsilon(\mathbf{v}) \mathrm{d} \Omega, \quad L(t)(\mathbf{v}):=\int_{\Omega} \mathbf{f}(t) \cdot \mathbf{v} \mathrm{d} \Omega+\int_{\Gamma_{N}} \mathbf{g}(t) \cdot \mathbf{v} \mathrm{d} \Gamma,
$$

for any $\mathbf{u}$ and $\mathbf{v}$ in $\mathbf{V}$, for all $t \in[0, T)$.

Remark 1.1. The (total) mechanical energy associated with the solution $\mathbf{u}$ of the dynamic contact Problems (1.1) and (1.2) is:

$$
E(t):=\frac{1}{2} \rho\|\dot{\mathbf{u}}(t)\|_{0, \Omega}^{2}+\frac{1}{2} a(\mathbf{u}(t), \mathbf{u}(t)), \quad \forall t \in[0, T] .
$$

Let us take $t \in[0, T]$. Formally, we get from (1.1), after multiplication by $\dot{\mathbf{u}}(t)$, integration by parts, with the boundary conditions on $\Gamma_{D T}, \Gamma_{N T}$ and the absence of friction:

$$
\underbrace{\int_{\Omega} \ddot{\mathbf{u}}(t) \cdot \dot{\mathbf{u}}(t) \mathrm{d} \Omega+\int_{\Omega} \boldsymbol{\sigma}(\mathbf{u}(t)): \boldsymbol{\varepsilon}(\dot{\mathbf{u}}(t)) \mathrm{d} \Omega}_{\frac{\mathrm{d}}{\mathrm{d} t} E(t)}-\int_{\Gamma_{C}} \sigma_{n}(\mathbf{u}(t)) \dot{\mathbf{u}}(t) d \Gamma=L(t)(\dot{\mathbf{u}}(t)) .
$$

Moreover, with the persistency condition $\sigma_{n}(\mathbf{u}(t)) \dot{\mathbf{u}}(t)=0$ (see, e.g., $[4,30,38]$ ) we end up with:

$$
\frac{\mathrm{d}}{\mathrm{d} t} E(t)=L(t)(\dot{\mathbf{u}}(t))
$$

In particular, when $L$ vanishes, we get energy conservation: $E(t)=E(0)$, for all $t \in[0, T]$. Note that in the $1 \mathrm{D}$ case (elastic bar), the energy conservation can be established rigorously (see [17], Lem. 2.5).

\section{Semi-Discretization in Space With a Nitsche-Based Finite Element method}

In this section we introduce our Nitsche-based FEM and carry out the well-posedness and stability analysis of the resulting space (semi-)discretization.

\subsection{Definition and preliminary results}

Let $\mathbf{V}^{h} \subset \mathbf{V}$ be a family of finite dimensional vector spaces (see [16]) indexed by $h$ coming from a family $\mathcal{T}^{h}$ of triangulations of the domain $\Omega\left(h=\max _{K \in \mathcal{T}^{h}} h_{K}\right.$ where $h_{K}$ is the diameter of the triangle $\left.K\right)$. The family of triangulations is supposed:

- regular, i.e., there exists $\sigma>0$ such that $\forall K \in \mathcal{T}^{h}, h_{K} / \rho_{K} \leq \sigma$ where $\rho_{K}$ denotes the radius of the inscribed ball in $K$, 
- conformal to the subdivision of the boundary into $\Gamma_{D}, \Gamma_{N}$ and $\Gamma_{C}$, which means that a face of an element $K \in$ $\mathcal{T}^{h}$ is not allowed to have simultaneous non-empty intersection with more than one part of the subdivision, - quasi-uniform, i.e., there exists $c>0$, such that, $\forall h>0, \forall K \in \mathcal{T}^{h}, h_{K} \geq c h$.

To fix ideas, we choose a standard Lagrange finite element method of degree $k$ with $k=1$ or $k=2$, i.e.:

$$
\mathbf{V}^{h}=\left\{\mathbf{v}^{h} \in\left(\mathscr{C}^{0}(\bar{\Omega})\right)^{d}: \mathbf{v}^{h}{ }_{K} \in\left(P_{k}(K)\right)^{d}, \forall K \in \mathcal{T}^{h}, \mathbf{v}^{h}=\mathbf{0} \text { on } \Gamma_{D}\right\} .
$$

However, the analysis would be similar for any $\mathscr{C}^{0}$-conforming finite element method.

Let us introduce the notation $[\cdot]_{+}$for the positive part of a scalar quantity $a \in \mathbb{R}$ :

$$
[a]_{+}=\left\{\begin{array}{cc}
a & \text { if } a>0 \\
0 & \text { otherwise }
\end{array}\right.
$$

The positive part has the following properties:

$$
a \leq[a]_{+}, \quad a[a]_{+}=[a]_{+}^{2}, \quad \forall a \in \mathbb{R} .
$$

Using (2.1), we recover a classical and useful property of the projection onto a closed convex set, i.e., for all $a, b \in \mathbb{R}:$

$$
\begin{aligned}
\left([a]_{+}-[b]_{+}\right)(a-b) & =a[a]_{+}+b[b]_{+}-b[a]_{+}-a[b]_{+} \\
& \geq[a]_{+}^{2}+[b]_{+}^{2}-2[a]_{+}[b]_{+} \\
& =\left([a]_{+}-[b]_{+}\right)^{2} \geq 0 .
\end{aligned}
$$

The Heaviside function will be noted $H(\cdot)$. We recall it can be defined as follows, for $a \in \mathbb{R}$ :

$$
H(a)= \begin{cases}1 & \text { if } a>0 \\ \frac{1}{2} & \text { if } a=0 \\ 0 & \text { if } a<0\end{cases}
$$

In fact it is a multivalued function for 0 , but we adopt the convention $H(0)=\frac{1}{2}$ to allow the property:

$$
H(a)+H(-a)=1, \quad \forall a \in \mathbb{R} .
$$

Note that conditions (2.1)-(2.3) can be straightforwardly extended to real valued functions. The derivation of a Nitsche-based method comes from a classical reformulation (see for instance [3]) of the contact conditions (1.2i)-(1.2iii):

$$
\sigma_{n}(\mathbf{u})=-\frac{1}{\gamma}\left[u_{n}-\gamma \sigma_{n}(\mathbf{u})\right]_{+},
$$

for any positive function $\gamma$ defined on $\Gamma_{C}$.

We consider in what follows that $\gamma=\gamma_{h}$ is a positive piecewise constant function on the contact interface $\Gamma_{C}$ which satisfies

$$
\left.\gamma_{h}\right|_{K \cap \Gamma_{C}}=\gamma_{0} h_{K},
$$

for every $K$ that has a non-empty intersection of dimension $d-1$ with $\Gamma_{C}$, and where $\gamma_{0}$ is a positive given constant. Note that the value of $\gamma_{h}$ on element intersections has no influence.

Let us introduce the discrete linear operator

$$
P_{\gamma_{h}}: \begin{aligned}
& \mathbf{V}^{h} \rightarrow \quad L^{2}\left(\Gamma_{C}\right) \\
& \mathbf{v}^{h} \mapsto v_{n}^{h}-\gamma_{h} \sigma_{n}\left(\mathbf{v}^{h}\right)
\end{aligned}
$$


and also the bilinear form:

$$
A_{\Theta \gamma_{h}}\left(\mathbf{u}^{h}, \mathbf{v}^{h}\right)=a\left(\mathbf{u}^{h}, \mathbf{v}^{h}\right)-\int_{\Gamma_{C}} \Theta \gamma_{h} \sigma_{n}\left(\mathbf{u}^{h}\right) \sigma_{n}\left(\mathbf{v}^{h}\right) \mathrm{d} \Gamma
$$

with $\Theta \in \mathbb{R}$ a fixed parameter.

Our space semi-discretized Nitsche-based method for unilateral contact problems in elastodynamics then reads:

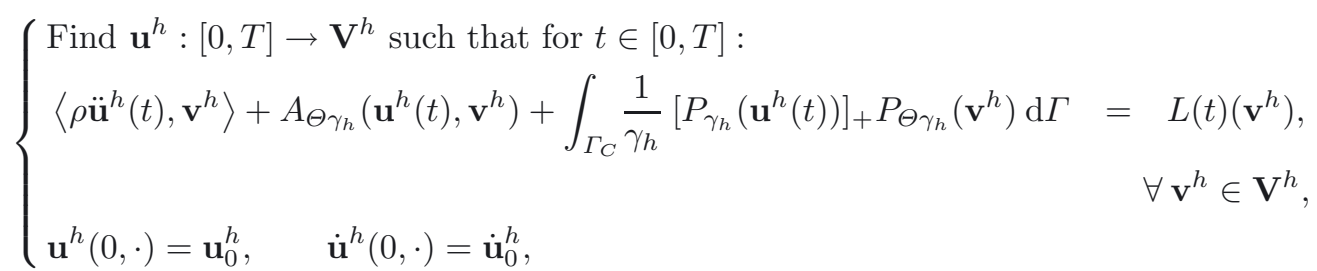

with $P_{\Theta \gamma_{h}}\left(\mathbf{v}_{h}\right):=v_{n}^{h}-\Theta \gamma_{h} \sigma_{n}\left(\mathbf{v}^{h}\right)$ and where $\mathbf{u}_{0}^{h}$ (resp. $\dot{\mathbf{u}}_{0}^{h}$ ) is an approximation in $\mathbf{V}^{h}$ of the initial displacement $\mathbf{u}_{0}$ (resp. the initial velocity $\dot{\mathbf{u}}_{0}$ ), for instance the Lagrange interpolant or the $L^{2}(\Omega)$ projection of $\mathbf{u}_{0}$ (resp. $\left.\dot{\mathbf{u}}_{0}\right)$. The notation $\langle\cdot, \cdot\rangle$ stands for the $L^{2}(\Omega)$ inner product.

Remark 2.1. Note that the parameter $\gamma_{0}$ in (2.5) is the inverse of the one generally used in the literature on Nitsche's method (see, e.g., $[27,45])$. This current choice is partly motivated by the analogy between Nitsche and the stabilized method of Barbosa and Hughes, in which $\gamma_{0}$ is the (small) stabilization parameter (see [44] and [13]).

Remark 2.2. We recall that in the case of mixed finite element methods, where a Lagrange multiplier stands for the contact stress, the space semi-discrete problem is a differential inclusion of the form (see, e.g., [33]):

$$
\begin{aligned}
\mathbf{M} \ddot{\mathbf{U}}+\mathbf{K U} & =\mathbf{L}+\mathbf{B}^{T} \boldsymbol{\Lambda}, \\
-\Lambda_{i} \in \partial I_{]-\infty ; 0]}\left((B U)_{i}\right), \quad \forall i & =1, \ldots, N_{C},
\end{aligned}
$$

where $(\mathbf{U}, \boldsymbol{\Lambda})$ are the column vectors associated with the solution (resp. displacement and Lagrange multiplier), $\mathbf{M}$, resp. $\mathbf{K}$, are the mass, resp. stiffness, matrices, $\mathbf{L}$ is the vector of external loads, $\mathbf{B}$ is the matrix of the normal trace operator and $N_{C}$ is the number of contact nodes. The indicator function of $\left.]-\infty ; 0\right]$ is denoted by $I_{]-\infty ; 0]}$ and its (multivalued) subgradient is $\partial I_{]-\infty ; 0]}$. The above differential inclusion is ill-posed since it can admit multiple solutions (see [33], Lem. 8, p. 89). For general references on differential inclusions, we refer the reader to, e.g., $[6,20]$.

In contrast with the standard (mixed) finite element semi-discretization, Nitsche's formulation leads to a well-posed (Lipschitz) system of differential equations, as it will be shown below. This feature is shared with the standard penalty method, the difference being that Nitsche's method remains consistent. Note that the standard (mixed) finite element semi-discretization is consistent as well as the singular dynamic method introduced in [42]. The mass redistribution method introduced in [34] is asymptotically consistent when $h$ vanishes.

In the rest of this section, we carry out the mathematical analysis of the method (2.6). First are defined some extra notations and introduced some preliminary results. We then show the consistency of the method in Section 2.2. The proof of well-posedness of problem (2.6) is carried out in Section 2.3. The energy conservation properties are studied in Section 2.4.

As usual for Nitsche's method (see e.g., $[7,44]$ ), we introduce the following mesh- and parameter-dependent scalar product in $\mathbf{V}^{h}$ :

$$
\left(\mathbf{v}^{h}, \mathbf{w}^{h}\right)_{\gamma_{h}}:=\left(\mathbf{v}^{h}, \mathbf{w}^{h}\right)_{1, \Omega}+\left(\gamma_{h}^{-\frac{1}{2}} v_{n}^{h}, \gamma_{h}^{-\frac{1}{2}} w_{n}^{h}\right)_{0, \Gamma_{C}} .
$$

We denote by $\|\cdot\|_{\gamma_{h}}:=(\cdot, \cdot)_{\gamma_{h}}^{\frac{1}{2}}$ the associated norm. 
We need first the following classical property, proven in [14] (see also [45], Lem. 2.1, p. 24, for the scalar case):

Lemma 2.3. There exists $C>0$, independent of the parameter $\gamma_{0}$ and of the mesh size $h$, such that:

$$
\left\|\gamma_{h}^{\frac{1}{2}} \sigma_{n}\left(\mathbf{v}^{h}\right)\right\|_{0, \Gamma_{C}}^{2} \leq C \gamma_{0}\left\|\mathbf{v}^{h}\right\|_{1, \Omega}^{2},
$$

for all $\mathbf{v}^{h} \in \mathbf{V}^{h}$.

The following inverse inequality will be of constant use, and for the proof, we refer for instance to ([22], Cor. 1.141, Rem. 1.143):

Lemma 2.4. Suppose that the mesh $\mathcal{T}^{h}$ is quasi-uniform, then for all $\mathbf{v}^{h} \in \mathbf{V}^{h}$ it holds:

$$
\left\|\mathbf{v}^{h}\right\|_{1, \Omega} \leq C h^{-1}\left\|\mathbf{v}^{h}\right\|_{0, \Omega} .
$$

\subsection{Consistency}

We show here the consistency of our Nitsche-based formulation (2.6) in the sense that, provided sufficient regularity conditions on the continuous solution $\mathbf{u}$ it is also solution to a space-time reformulation of (2.6). The space-time formulation is needed to avoid assumptions on the regularity of $\mathbf{u}$ that would be too restrictive.

Proposition 2.5. The Nitsche-based method for contact is consistent in the following sense: suppose that the solution $\mathbf{u}$ of $(1.3)$ is in $\left(H^{\frac{3}{2}+\nu}\left(\Omega_{T}\right)\right)^{d}$ (with $\left.0<\nu<\frac{1}{2}\right)$, then $\mathbf{u}$ is also solution of

$$
\begin{aligned}
& -\int_{\Omega} \rho \dot{\mathbf{u}}_{0} \cdot \mathbf{v}^{h}(0, \cdot) \mathrm{d} \Omega-\int_{\Omega_{T}} \rho \dot{\mathbf{u}} \cdot \dot{\mathbf{v}}^{h} \mathrm{~d} \Omega \mathrm{d} t+\int_{\Omega_{T}} \boldsymbol{\sigma}(\mathbf{u}): \varepsilon\left(\mathbf{v}^{h}\right) \mathrm{d} \Omega \mathrm{d} t \\
& -\int_{\Gamma_{C T}} \Theta \gamma_{h} \sigma_{n}(\mathbf{u}) \sigma_{n}\left(\mathbf{v}^{h}\right) \mathrm{d} \Gamma \mathrm{d} t+\int_{\Gamma_{C T}} \frac{1}{\gamma_{h}}\left[P_{\gamma_{h}}(\mathbf{u})\right]_{+} P_{\Theta \gamma_{h}}\left(\mathbf{v}^{h}\right) \mathrm{d} \Gamma \mathrm{d} t \\
& =\int_{\Omega_{T}}^{C T} \mathbf{f} \cdot \mathbf{v}^{h} \mathrm{~d} \Omega \mathrm{d} t+\int_{\Gamma_{N T}} \mathbf{g} \cdot \mathbf{v}^{h} \mathrm{~d} \Gamma \mathrm{d} t \quad \forall \mathbf{v}^{h} \in \mathcal{D}([0, T)) \otimes \mathbf{V}^{h},
\end{aligned}
$$

where $\mathcal{D}([0, T)) \otimes \mathbf{V}^{h}=\mathscr{L}\left\{\mathbf{w}: \Omega_{T} \rightarrow \mathbb{R}^{d}: \mathbf{w}(t, x)=f(t) \mathbf{g}(x), f \in \mathcal{D}([0, T)), \mathbf{g} \in \mathbf{V}^{h}\right\}$, the notation $\mathscr{L} E$ standing for all the finite linear combinations of elements in $E$, and $\mathcal{D}(I)$ being the vectorial space of $\mathcal{C}^{\infty}$ real functions with compact support in $I$.

Remark 2.6. Note the difference between Nitsche's formulation (2.6), which involves integration on $\Omega$ at each time $t$, and the equation of the Proposition 2.5, which is a space-time formulation on the whole cylinder $\Omega_{T}$. The regularity assumptions on $\mathbf{u}$ are too weak so that we can substitute to this latter a space formulation at almost every time $t$. When $\mathbf{u} \in\left(H^{\frac{3}{2}+\nu}\left(\Omega_{T}\right)\right)^{d}$ it implies only $\mathbf{u}(t) \in\left(H^{1+\nu}(\Omega)\right)^{d}, \dot{\mathbf{u}} \in\left(H^{\frac{1}{2}+\nu}\left(\Omega_{T}\right)\right)^{d}$, $\dot{\mathbf{u}}(t) \in\left(H^{\nu}(\Omega)\right)^{d}$ and $\sigma(\mathbf{u}) \in\left(H^{\frac{1}{2}+\nu}\left(\Omega_{T}\right)\right)^{d \times d}$. It has to be compatible with the impact phenomenon, in which $\dot{\mathbf{u}}$ is expected to be discontinuous in time and $\ddot{\mathbf{u}}(t) \notin L^{2}(\Omega)$.

Proof. Let $\mathbf{u}$ be the solution of (1.3). Due to the assumed regularity and Green's formula it is equivalently solution of:

$$
\left\{\begin{array}{l}
-\int_{\Omega} \rho \dot{\mathbf{u}}_{0} \cdot \mathbf{v}(0, \cdot) \mathrm{d} \Omega-\int_{\Omega_{T}} \rho \dot{\mathbf{u}} \cdot \dot{\mathbf{v}} \mathrm{d} \Omega \mathrm{d} t+\int_{\Omega_{T}} \boldsymbol{\sigma}(\mathbf{u}): \varepsilon(\mathbf{v}) \mathrm{d} \Omega \mathrm{d} t-\int_{\Gamma_{C T}} \sigma_{n}(\mathbf{u}) v_{n} \mathrm{~d} \Gamma \mathrm{d} t \\
=\int_{\Omega_{T}} \mathbf{f} \cdot \mathbf{v} \mathrm{d} \Omega \mathrm{d} t+\int_{\Gamma_{N T}} \mathbf{g} \cdot \mathbf{v} \mathrm{d} \Gamma \mathrm{d} t \\
\text { for all } \mathbf{v} \in \mathcal{W} \text { such that } \mathbf{v}=\mathbf{0} \text { in a neighborhood of } T,
\end{array}\right.
$$


with contact conditions (1.2i)-(1.2iii) verified a.e. on $\Gamma_{C T}$. The regularity assumption $\mathbf{u} \in\left(H^{\frac{3}{2}+\nu}\left(\Omega_{T}\right)\right)^{d}(\nu>0)$ yields $\sigma_{n}(\mathbf{u}) \in H^{\nu}\left(\Gamma_{C T}\right) \subset L^{2}\left(\Gamma_{C T}\right)$ so that in formula (2.9), the integral term on $\Gamma_{C T}$ is correctly defined, and the contact conditions hold in $L^{2}\left(\Gamma_{C T}\right)$.

Take now $\mathbf{v}^{h} \in \mathcal{D}([0, T)) \otimes \mathbf{V}^{h}$, in particular $\mathbf{v}^{h} \in \mathcal{W}$ and $\mathbf{v}^{h}=0$ in a neighborhood of $T$. The inner product $\sigma_{n}(\mathbf{u}) \sigma_{n}\left(\mathbf{v}^{h}\right)$ in $L^{2}\left(\Gamma_{C T}\right)$ is meaningful, so we can rewrite (2.9) as follows:

$$
\left\{\begin{array}{l}
-\int_{\Omega} \rho \dot{\mathbf{u}}_{0} \cdot \mathbf{v}^{h}(0, \cdot) \mathrm{d} \Omega-\int_{\Omega_{T}} \rho \dot{\mathbf{u}} \cdot \dot{\mathbf{v}}^{h} \mathrm{~d} \Omega \mathrm{d} t+\int_{\Omega_{T}} \boldsymbol{\sigma}(\mathbf{u}): \varepsilon\left(\mathbf{v}^{h}\right) \mathrm{d} \Omega \mathrm{d} t-\int_{\Gamma_{C T}} \Theta \gamma_{h} \sigma_{n}(\mathbf{u}) \sigma_{n}\left(\mathbf{v}^{h}\right) \mathrm{d} \Gamma \mathrm{d} t \\
-\int_{\Gamma_{C T}} \sigma_{n}(\mathbf{u})\left(v_{n}-\Theta \gamma_{h} \sigma_{n}\left(\mathbf{v}^{h}\right)\right) \mathrm{d} \Gamma \mathrm{d} t=\int_{\Omega_{T}} \mathbf{f} \cdot \mathbf{v}^{h} \mathrm{~d} \Omega \mathrm{d} t+\int_{\Gamma_{N T}} \mathbf{g} \cdot \mathbf{v}^{h} \mathrm{~d} \Gamma \mathrm{d} t \\
\text { for all } \mathbf{v}^{h} \in \mathcal{D}([0, T)) \otimes \mathbf{V}^{h} .
\end{array}\right.
$$

With the reformulation of contact conditions (2.4), which makes sense in $L^{2}\left(\Gamma_{C T}\right)$, and the definition of $P_{\gamma_{h}}$, the proof is finished.

Remark 2.7. The regularity $\mathbf{u} \in\left(H^{\frac{3}{2}+\nu}\left(\Omega_{T}\right)\right)^{d}$ in the consistency result could probably be weakened to $\sigma_{n}(\mathbf{u}) \in L^{2}\left(\Gamma_{C T}\right)$ since the condition $\mathbf{f} \in \mathscr{C}^{0}\left([0, T] ;\left(L^{2}(\Omega)\right)^{d}\right)$ is sufficient to give a sense to a Green-like formula on $\Omega_{T}$.

\subsection{Well-posedness}

In order to prove well-posedness we reformulate (2.6) as a system of (non-linear) second-order differential equations. To this purpose, using Riesz's representation theorem in $\left(\mathbf{V}^{h},(\cdot, \cdot)_{\gamma_{h}}\right)$ we first introduce the mass operator $\mathbf{M}^{h}: \mathbf{V}^{h} \rightarrow \mathbf{V}^{h}$, which is defined for all $\mathbf{v}^{h}, \mathbf{w}^{h} \in \mathbf{V}^{h}$ by

$$
\left(\mathbf{M}^{h} \mathbf{v}^{h}, \mathbf{w}^{h}\right)_{\gamma_{h}}=\left\langle\rho \mathbf{v}^{h}, \mathbf{w}^{h}\right\rangle
$$

Still using Riesz's representation theorem, we define the (non-linear) operator $\mathbf{B}^{h}: \mathbf{V}^{h} \rightarrow \mathbf{V}^{h}$, by means of the formula

$$
\left(\mathbf{B}^{h} \mathbf{v}^{h}, \mathbf{w}^{h}\right)_{\gamma_{h}}=A_{\Theta \gamma_{h}}\left(\mathbf{v}^{h}, \mathbf{w}^{h}\right)+\int_{\Gamma_{C}} \frac{1}{\gamma_{h}}\left[P_{\gamma_{h}}\left(\mathbf{v}^{h}\right)\right]_{+} P_{\Theta \gamma_{h}}\left(\mathbf{w}^{h}\right) \mathrm{d} \Gamma,
$$

for all $\mathbf{v}^{h}, \mathbf{w}^{h} \in \mathbf{V}^{h}$. Finally, we denote by $\mathbf{L}^{h}(t)$ the vector in $\mathbf{V}^{h}$ such that, for all $t \in[0, T]$ and for every $\mathbf{w}^{h}$ in $\mathbf{V}^{h}$ :

$$
\left(\mathbf{L}^{h}(t), \mathbf{w}^{h}\right)_{\gamma_{h}}=L(t)\left(\mathbf{w}^{h}\right) .
$$

Remark that, due to the assumptions on $\mathbf{f}$ and $\mathbf{g}, \mathbf{L}^{h}$ is continuous from $[0, T]$ onto $\left(\mathbf{V}_{h},\|\cdot\|_{\gamma_{h}}\right)$.

With the above notation, problem (2.6) reads:

$$
\left\{\begin{array}{l}
\text { Find } \mathbf{u}^{h}:[0, T] \rightarrow \mathbf{V}^{h} \text { such that for } t \in[0, T]: \\
\mathbf{M}^{h} \ddot{\mathbf{u}}^{h}(t)+\mathbf{B}^{h} \mathbf{u}^{h}(t)=\mathbf{L}^{h}(t), \\
\mathbf{u}^{h}(0, \cdot)=\mathbf{u}_{0}^{h}, \quad \dot{\mathbf{u}}^{h}(0, \cdot)=\dot{\mathbf{u}}_{0}^{h} .
\end{array}\right.
$$

We then show that problem (2.6) (or equivalently problem (2.10)) is well-posed.

Theorem 2.8. The operator $\mathbf{B}^{h}$ is Lipschitz-continuous in the following sense: there exists a constant $C>0$, independent of $h, \Theta$ and $\gamma_{0}$ such that, for all $\mathbf{v}_{1}^{h}, \mathbf{v}_{2}^{h} \in \mathbf{V}^{h}$ :

$$
\left\|\mathbf{B}^{h} \mathbf{v}_{1}^{h}-\mathbf{B}^{h} \mathbf{v}_{2}^{h}\right\|_{\gamma_{h}} \leq C\left(1+\gamma_{0}\right)(1+|\Theta|)\left\|\mathbf{v}_{1}^{h}-\mathbf{v}_{2}^{h}\right\|_{\gamma_{h}} .
$$

As a consequence, for every value of $\Theta \in \mathbb{R}$ and $\gamma_{0}>0$, problem (2.6) admits one unique solution $\mathbf{u}^{h} \in$ $\mathscr{C}^{2}\left([0, T], \mathbf{V}^{h}\right)$. 
Proof. Let us pick $\mathbf{v}_{1}^{h}, \mathbf{v}_{2}^{h}, \mathbf{w}^{h} \in \mathbf{V}^{h}$, then:

$$
\begin{aligned}
& \left|\left(\mathbf{B}^{h} \mathbf{v}_{1}^{h}-\mathbf{B}^{h} \mathbf{v}_{2}^{h}, \mathbf{w}^{h}\right)_{\gamma_{h}}\right| \\
= & \left|A_{\Theta \gamma_{h}}\left(\mathbf{v}_{1}^{h}-\mathbf{v}_{2}^{h}, \mathbf{w}^{h}\right)+\int_{\Gamma_{C}} \frac{1}{\gamma_{h}}\left(\left[P_{\gamma_{h}}\left(\mathbf{v}_{1}^{h}\right)\right]_{+}-\left[P_{\gamma_{h}}\left(\mathbf{v}_{2}^{h}\right)\right]_{+}\right) P_{\Theta \gamma_{h}}\left(\mathbf{w}^{h}\right) \mathrm{d} \Gamma\right| \\
\leq & C\left(1+|\Theta| \gamma_{0}\right)\left\|\mathbf{v}_{1}^{h}-\mathbf{v}_{2}^{h}\right\|_{1, \Omega}\left\|\mathbf{w}^{h}\right\|_{1, \Omega}+\int_{\Gamma_{C}} \frac{1}{\gamma_{h}}\left|\left[P_{\gamma_{h}}\left(\mathbf{v}_{1}^{h}\right)\right]_{+}-\left[P_{\gamma_{h}}\left(\mathbf{v}_{2}^{h}\right)\right]_{+}\right|\left|P_{\Theta \gamma_{h}}\left(\mathbf{w}^{h}\right)\right| \mathrm{d} \Gamma,
\end{aligned}
$$

as the estimate (2.7) yields $\left\|A_{\Theta \gamma_{h}}\right\| \leq C\left(1+|\Theta| \gamma_{0}\right)$.

With the inequality $\left|[a]_{+}-[b]_{+}\right| \leq|a-b|$, for all $a, b \in \mathbb{R}$, and using the linearity of $P_{\gamma_{h}}$, we remark that:

$$
\begin{aligned}
& \int_{\Gamma_{C}} \frac{1}{\gamma_{h}}\left|\left[P_{\gamma_{h}}\left(\mathbf{v}_{1}^{h}\right)\right]_{+}-\left[P_{\gamma_{h}}\left(\mathbf{v}_{2}^{h}\right)\right]_{+}\right|\left|P_{\Theta \gamma_{h}}\left(\mathbf{w}^{h}\right)\right| \mathrm{d} \Gamma \\
\leq & \int_{\Gamma_{C}} \frac{1}{\gamma_{h}}\left|P_{\gamma_{h}}\left(\mathbf{v}_{1}^{h}-\mathbf{v}_{2}^{h}\right)\right|\left|P_{\Theta \gamma_{h}}\left(\mathbf{w}^{h}\right)\right| \mathrm{d} \Gamma \\
\leq & \left\|\gamma_{h}{ }^{-\frac{1}{2}} P_{\gamma_{h}}\left(\mathbf{v}_{1}^{h}-\mathbf{v}_{2}^{h}\right)\right\|_{0, \Gamma_{C}}\left\|\gamma_{h}{ }^{-\frac{1}{2}} P_{\Theta \gamma_{h}}\left(\mathbf{w}^{h}\right)\right\|_{0, \Gamma_{C}} \\
\leq & \left(\left\|\gamma_{h}{ }^{-\frac{1}{2}}\left(v_{1, n}^{h}-v_{2, n}^{h}\right)\right\|_{0, \Gamma_{C}}+\left\|\gamma_{h}^{\frac{1}{2}} \sigma_{n}\left(\mathbf{v}_{1}^{h}-\mathbf{v}_{2}^{h}\right)\right\|_{0, \Gamma_{C}}\right)\left(\left\|\gamma_{h}^{-\frac{1}{2}} w_{n}^{h}\right\|_{0, \Gamma_{C}}+|\Theta|\left\|\gamma_{h}^{\frac{1}{2}} \sigma_{n}\left(\mathbf{w}^{h}\right)\right\|_{0, \Gamma_{C}}\right) \\
\leq & \left(\left\|\gamma_{h}{ }^{-\frac{1}{2}}\left(v_{1, n}^{h}-v_{2, n}^{h}\right)\right\|_{0, \Gamma_{C}}+C \gamma_{0}^{\frac{1}{2}}\left\|\mathbf{v}_{1}^{h}-\mathbf{v}_{2}^{h}\right\|_{1, \Omega}\right)\left(\left\|\gamma_{h}^{-\frac{1}{2}} w_{n}^{h}\right\|_{0, \Gamma_{C}}+C|\Theta| \gamma_{0}^{\frac{1}{2}}\left\|\mathbf{w}^{h}\right\|_{1, \Omega}\right) .
\end{aligned}
$$

In the last lines, we used the Cauchy-Schwarz inequality, the triangular inequality and the estimate (2.7). Taking this bound into account, we now combine the above estimations to obtain:

$$
\begin{aligned}
& \left|\left(\mathbf{B}^{h} \mathbf{v}_{1}^{h}-\mathbf{B}^{h} \mathbf{v}_{2}^{h}, \mathbf{w}^{h}\right)_{\gamma_{h}}\right| \\
\leq & C\left(1+|\Theta| \gamma_{0}\right)\left\|\mathbf{v}_{1}^{h}-\mathbf{v}_{2}^{h}\right\|_{1, \Omega}\left\|\mathbf{w}^{h}\right\|_{1, \Omega} \\
& +\left(C \gamma_{0}^{\frac{1}{2}}\left\|\mathbf{v}_{1}^{h}-\mathbf{v}_{2}^{h}\right\|_{1, \Omega}+\left\|\gamma_{h}{ }^{-\frac{1}{2}}\left(v_{1, n}^{h}-v_{2, n}^{h}\right)\right\|_{0, \Gamma_{C}}\right)\left(C|\Theta| \gamma_{0}^{\frac{1}{2}}\left\|\mathbf{w}^{h}\right\|_{1, \Omega}+\left\|\gamma_{h}^{-\frac{1}{2}} w_{n}^{h}\right\|_{0, \Gamma_{C}}\right) \\
\leq & C\left(1+|\Theta| \gamma_{0}\right)\left\|\mathbf{v}_{1}^{h}-\mathbf{v}_{2}^{h}\right\|_{1, \Omega}\left\|\mathbf{w}^{h}\right\|_{1, \Omega} \\
& +C\left(1+\gamma_{0}^{\frac{1}{2}}\right)\left(1+|\Theta| \gamma_{0}^{\frac{1}{2}}\right)\left(\left\|\mathbf{v}_{1}^{h}-\mathbf{v}_{2}^{h}\right\|_{1, \Omega}+\left\|\gamma_{h}^{-\frac{1}{2}}\left(v_{1, n}^{h}-v_{2, n}^{h}\right)\right\|_{0, \Gamma_{C}}\right)\left(\left\|\mathbf{w}^{h}\right\|_{1, \Omega}+\left\|\gamma_{h}{ }^{-\frac{1}{2}} w_{n}^{h}\right\|_{0, \Gamma_{C}}\right) \\
\leq & C\left(1+\gamma_{0}\right)(1+|\Theta|) \underbrace{\left(\left\|\mathbf{v}_{1}^{h}-\mathbf{v}_{2}^{h}\right\|_{1, \Omega}^{2}+\left\|\gamma_{h}^{-\frac{1}{2}}\left(v_{1, n}^{h}-v_{2, n}^{h}\right)\right\|_{0, \Gamma_{C}}^{2}\right)^{\frac{1}{2}}}_{\left\|\mathbf{v}_{1}^{h}-\mathbf{v}_{2}^{h}\right\|_{\gamma_{h}}} \underbrace{\left(\left\|\mathbf{w}^{h}\right\|_{1, \Omega}^{2}+\left\|\gamma_{h}{ }^{-\frac{1}{2}} w_{n}^{h}\right\|_{0, \Gamma_{C}}^{2}\right)^{\frac{1}{2}}}_{\left\|\mathbf{w}^{h}\right\|_{\gamma_{h}}} .
\end{aligned}
$$

It results that

$$
\left\|\mathbf{B}^{h} \mathbf{v}_{1}^{h}-\mathbf{B}^{h} \mathbf{v}_{2}^{h}\right\|_{\gamma_{h}}=\sup _{\mathbf{w}^{h} \in \mathbf{V}^{h}} \frac{\left|\left(\mathbf{B}^{h} \mathbf{v}_{1}^{h}-\mathbf{B}^{h} \mathbf{v}_{2}^{h}, \mathbf{w}^{h}\right)_{\gamma_{h}}\right|}{\left\|\mathbf{w}^{h}\right\|_{\gamma_{h}}} \leq C\left(1+\gamma_{0}\right)(1+|\Theta|)\left\|\mathbf{v}_{1}^{h}-\mathbf{v}_{2}^{h}\right\|_{\gamma_{h}} .
$$

This proves the first assertion of the theorem.

Then we recast (2.10) in the canonical form of a first-order system:

$$
\frac{\mathrm{d}}{\mathrm{d} t} \mathbf{x}^{h}(t)=\mathbf{F}^{h}\left(t, \mathbf{x}^{h}(t)\right), \quad \mathbf{x}^{h}(0)=\mathbf{x}_{0}^{h}
$$

where:

$$
\mathbf{x}^{h}(t):=\left[\begin{array}{c}
\dot{\mathbf{u}}^{h} \\
\mathbf{u}^{h}
\end{array}\right](t), \quad \mathbf{x}_{0}^{h}:=\left[\begin{array}{c}
\dot{\mathbf{u}}_{0}^{h} \\
\mathbf{u}_{0}^{h},
\end{array}\right], \quad \mathbf{F}^{h}\left(t, \mathbf{x}^{h}(t)\right):=\left[\begin{array}{c}
\left(\mathbf{M}^{h}\right)^{-1}\left(\mathbf{L}^{h}(t)-\mathbf{B}^{h} \mathbf{u}^{h}(t)\right) \\
\dot{\mathbf{u}}^{h}(t)
\end{array}\right]
$$


It holds for arbitrary $t \in[0, T]$ and $\mathbf{x}_{1}^{h}, \mathbf{x}_{2}^{h} \in\left(\mathbf{V}^{h}\right)^{2}$ :

$$
\left\|\mathbf{F}^{h}\left(t, \mathbf{x}_{1}^{h}\right)-\mathbf{F}^{h}\left(t, \mathbf{x}_{2}^{h}\right)\right\|_{\gamma_{h} \times \gamma_{h}}^{2}=\left\|\left(\mathbf{M}^{h}\right)^{-1}\left(\mathbf{B}^{h} \mathbf{u}_{2}^{h}-\mathbf{B}^{h} \mathbf{u}_{1}^{h}\right)\right\|_{\gamma_{h}}^{2}+\left\|\dot{\mathbf{u}}_{1}^{h}-\dot{\mathbf{u}}_{2}^{h}\right\|_{\gamma_{h}}^{2},
$$

where $\|\cdot\|_{\gamma_{h} \times \gamma_{h}}$ denotes the product norm on $\left(\mathbf{V}^{h}\right)^{2}$.

From the estimates (A.1) (see Appendix A), (2.11) and re-arranging the terms we get:

$$
\begin{aligned}
\left\|\left(\mathbf{M}^{h}\right)^{-1}\left(\mathbf{B}^{h} \mathbf{u}_{2}^{h}-\mathbf{B}^{h} \mathbf{u}_{1}^{h}\right)\right\|_{\gamma_{h}} & \leq\left\|\left(\mathbf{M}^{h}\right)^{-1}\right\|_{\gamma_{h}}\left\|\mathbf{B}^{h} \mathbf{u}_{2}^{h}-\mathbf{B}^{h} \mathbf{u}_{1}^{h}\right\|_{\gamma_{h}} \\
& \leq C \rho^{-1}\left(1+\gamma_{0}^{-1}\right) h^{-2}\left(1+\gamma_{0}\right)(1+|\Theta|)\left\|\mathbf{u}_{2}^{h}-\mathbf{u}_{1}^{h}\right\|_{\gamma_{h}} \\
& \leq C \rho^{-1} h^{-2}(1+|\Theta|)\left(1+\gamma_{0}+\gamma_{0}^{-1}\right)\left\|\mathbf{u}_{2}^{h}-\mathbf{u}_{1}^{h}\right\|_{\gamma_{h}} .
\end{aligned}
$$

The second assertion of the theorem is a consequence of the Lipschitz-continuity of $\mathbf{F}^{h}$ and of the Cauchy-Lipschitz (Picard-Lindelöf) theorem.

Remark 2.9. Note that, conversely to the static case (see [12-14]) and the fully-discrete case (see Sect. 3.2), there is no condition on $\gamma_{0}$ for the space (semi-)discretization, which remains well-posed even if $\gamma_{0}$ is large.

\subsection{Energy estimates and stability}

This section is devoted to energy estimates which are counterparts of the equation (1.4), in the semi-discretized case. Let us define the discrete energy as follows:

$$
E^{h}(t):=\frac{1}{2} \rho\left\|\dot{\mathbf{u}}^{h}(t)\right\|_{0, \Omega}^{2}+\frac{1}{2} a\left(\mathbf{u}^{h}(t), \mathbf{u}^{h}(t)\right),
$$

which is associated to the solution $\mathbf{u}^{h}(t)$ to Problem (2.6). Note that this is the direct transposition of the mechanical energy $E(t)$ for the continuous system. Set also

$$
E_{\Theta}^{h}(t):=E^{h}(t)-\frac{\Theta}{2}\left[\left\|\gamma_{h}^{\frac{1}{2}} \sigma_{n}\left(\mathbf{u}^{h}(t)\right)\right\|_{0, \Gamma_{C}}^{2}-\left\|\gamma_{h}{ }^{-\frac{1}{2}}\left[P_{\gamma_{h}}\left(\mathbf{u}^{h}(t)\right)\right]_{+}\right\|_{0, \Gamma_{C}}^{2}\right]:=E^{h}(t)-\Theta R^{h}(t),
$$

which corresponds to a modified energy in which a consistent term is added. This term denoted $R^{h}(t)$ represents, roughly speaking, the nonfulfillment of the contact condition (2.4) by $\mathbf{u}^{h}$.

Theorem 2.10. Suppose that the system associated to (1.3) is conservative, i.e., that $L(t) \equiv 0$ for all $t \in[0, T]$. The solution $\mathbf{u}^{h}$ of (2.6) then satisfies the following identity:

$$
\frac{\mathrm{d}}{\mathrm{d} t} E_{\Theta}^{h}(t)=(\Theta-1) \int_{\Gamma_{C}} \frac{1}{\gamma_{h}}\left[P_{\gamma_{h}}\left(\mathbf{u}^{h}(t)\right)\right]_{+} \dot{u}_{n}^{h}(t) \mathrm{d} \Gamma .
$$

In particular, when $\Theta=1$, we get for any $t \in[0, T]: E_{1}^{h}(t)=E_{1}^{h}(0)$.

Corollary 2.11. With the same assumptions as in the previous theorem, the variation of the discrete elastic energy $E^{h}(t)$ only comes from the non fulfillment of the exact contact conditions at the discrete level. More precisely:

$$
E^{h}(t)=E^{h}(0)+\Theta\left(R^{h}(t)-R^{h}(0)\right)+(\Theta-1) \int_{0}^{t} \int_{\Gamma_{C}} \frac{1}{\gamma_{h}}\left[P_{\gamma_{h}}\left(\mathbf{u}^{h}(s)\right)\right]_{+} \dot{u}_{n}^{h}(s) \mathrm{d} \Gamma \mathrm{d} s .
$$

Proof. Let us suppose that $L(t) \equiv 0$ for all $t \in[0, T]$. We take $\mathbf{v}^{h}=\dot{\mathbf{u}}^{h}(t) \in \mathbf{V}^{h}$ as a test function in (2.6). So we obtain (to lighten the notations we write $\mathbf{u}^{h}$ instead of $\mathbf{u}^{h}(t)$ ):

$$
\rho\left\langle\ddot{\mathbf{u}}^{h}, \dot{\mathbf{u}}^{h}\right\rangle+a\left(\mathbf{u}^{h}, \dot{\mathbf{u}}^{h}\right)-\int_{\Gamma_{C}} \Theta \gamma_{h} \sigma_{n}\left(\mathbf{u}^{h}\right) \sigma_{n}\left(\dot{\mathbf{u}}^{h}\right) \mathrm{d} \Gamma+\int_{\Gamma_{C}} \frac{1}{\gamma_{h}}\left[P_{\gamma_{h}}\left(\mathbf{u}^{h}\right)\right]_{+} P_{\Theta \gamma_{h}}\left(\dot{\mathbf{u}}^{h}\right) \mathrm{d} \Gamma=0 .
$$


For the first two terms:

$$
\rho\left\langle\ddot{\mathbf{u}}^{h}, \dot{\mathbf{u}}^{h}\right\rangle+a\left(\mathbf{u}^{h}, \dot{\mathbf{u}}^{h}\right)=\frac{\mathrm{d}}{\mathrm{d} t} E^{h}(t) .
$$

By using the definition of $P_{\Theta \gamma_{h}}$ and re-arranging the terms, we get:

$$
\begin{aligned}
\frac{\mathrm{d}}{\mathrm{d} t} E^{h}(t) & =\Theta \int_{\Gamma_{C}} \gamma_{h} \sigma_{n}\left(\mathbf{u}^{h}\right) \sigma_{n}\left(\dot{\mathbf{u}}^{h}\right) \mathrm{d} \Gamma-\Theta \int_{\Gamma_{C}} \frac{1}{\gamma_{h}}\left[P_{\gamma_{h}}\left(\mathbf{u}^{h}\right)\right]_{+} P_{\gamma_{h}}\left(\dot{\mathbf{u}}^{h}\right) \mathrm{d} \Gamma-(1-\Theta) \int_{\Gamma_{C}} \frac{1}{\gamma_{h}}\left[P_{\gamma_{h}}\left(\mathbf{u}^{h}\right)\right]_{+} \dot{u}_{n}^{h} \mathrm{~d} \Gamma \\
& =\frac{\Theta}{2} \frac{\mathrm{d}}{\mathrm{d} t}\left\|\gamma_{h}{ }^{\frac{1}{2}} \sigma_{n}\left(\mathbf{u}^{h}\right)\right\|_{0, \Gamma_{C}}^{2}-\frac{\Theta}{2} \frac{\mathrm{d}}{\mathrm{d} t}\left\|\gamma_{h}{ }^{-\frac{1}{2}}\left[P_{\gamma_{h}}\left(\mathbf{u}^{h}\right)\right]_{+}\right\|_{0, \Gamma_{C}}^{2}-(1-\Theta) \int_{\Gamma_{C}} \frac{1}{\gamma_{h}}\left[P_{\gamma_{h}}\left(\mathbf{u}^{h}\right)\right]_{+} \dot{u}_{n}^{h} \mathrm{~d} \Gamma .
\end{aligned}
$$

In the last line, we made use of the following formula, for $\phi: \mathbb{R} \rightarrow \mathbb{R}$ :

$$
\frac{1}{2} \frac{\mathrm{d}}{\mathrm{d} t}[\phi(t)]_{+}^{2}=[\phi(t)]_{+} \frac{\mathrm{d}}{\mathrm{d} t}[\phi(t)]_{+}=[\phi(t)]_{+} H(\phi(t)) \frac{\mathrm{d}}{\mathrm{d} t} \phi(t)=[\phi(t)]_{+} \frac{\mathrm{d}}{\mathrm{d} t} \phi(t),
$$

with the property $[a]_{+} H(a)=[a]_{+}(a \in \mathbb{R})$. That concludes the proof of the theorem. The proof of the corollary is straightforward.

Remark 2.12. The mechanical energy $E^{h}(t)$ can fluctuate whenever the discrete persistency condition $\left[P_{\gamma_{h}}\left(\mathbf{u}^{h}(t)\right)\right]_{+} \dot{u}_{n}^{h}(t)=0$ or the discrete contact condition $\sigma_{n}\left(\mathbf{u}^{h}(t)\right)+\gamma_{h}{ }^{-1}\left[P_{\gamma_{h}}\left(\mathbf{u}^{h}(t)\right)\right]_{+}=0$ are not satisfied.

\section{Fully Discrete formulations}

In this section we fully discretize the dynamic contact problem by combining Nitsche's method with some classical schemes ( $\theta$-scheme, Newmark) as well as a new hybrid scheme. We study the well-posedness of the schemes.

Let $\tau>0$ be the time-step, and consider a uniform discretization of the time interval $[0, T]:\left(t^{0}, \ldots, t^{N}\right)$, with $t^{n}=n \tau, n=0, \ldots, N$. Let $\theta \in[0,1]$, we use the notation:

$$
\mathbf{x}^{h, n+\theta}=(1-\theta) \mathbf{x}^{h, n}+\theta \mathbf{x}^{h, n+1}
$$

for arbitrary quantities $\mathbf{x}^{h, n}, \mathbf{x}^{h, n+1} \in \mathbf{V}^{h}$. Hereafter we denote by $\mathbf{u}^{h, n}$ (resp. $\dot{\mathbf{u}}^{h, n}$ and $\ddot{\mathbf{u}}^{h, n}$ ) the resulting discretized displacement (resp. velocity and acceleration) at time-step $t^{n}$.

\subsection{Proposed time-marching schemes}

\subsubsection{A $\theta$-scheme}

We semi-discretize in time problem (2.6) using a $\theta$-scheme, which parameter is $\theta \in[0,1]$. For $n \geq 0$, the fully discretized problem reads:

$$
\left\{\begin{array}{l}
\text { Find } \mathbf{u}^{h, n+1}, \dot{\mathbf{u}}^{h, n+1}, \ddot{\mathbf{u}}^{h, n+1} \in \mathbf{V}^{h} \text { such that: } \\
\mathbf{u}^{h, n+1}=\mathbf{u}^{h, n}+\tau \dot{\mathbf{u}}^{h, n+\theta} \\
\dot{\mathbf{u}}^{h, n+1}=\dot{\mathbf{u}}^{h, n}+\tau \ddot{\mathbf{u}}^{h, n+\theta}, \\
\left\langle\rho \ddot{\mathbf{u}}^{h, n+1}, \mathbf{v}^{h}\right\rangle+A_{\Theta \gamma_{h}}\left(\mathbf{u}^{h, n+1}, \mathbf{v}^{h}\right)+\int_{\Gamma_{C}} \frac{1}{\gamma_{h}}\left[P_{\gamma_{h}}\left(\mathbf{u}^{h, n+1}\right)\right]_{+} P_{\Theta \gamma_{h}}\left(\mathbf{v}^{h}\right) \mathrm{d} \Gamma=L^{n+1}\left(\mathbf{v}^{h}\right), \\
\quad \forall \mathbf{v}^{h} \in \mathbf{V}^{h},
\end{array}\right.
$$

with initial conditions $\mathbf{u}^{h, 0}=\mathbf{u}_{0}^{h}, \dot{\mathbf{u}}^{h, 0}=\dot{\mathbf{u}}_{0}^{h}, \ddot{\mathbf{u}}^{h, 0}=\ddot{\mathbf{u}}_{0}^{h}$ (see Rem. 3.2 below), and where $L^{n+1}(\cdot)=L\left(t^{n+1}\right)(\cdot)$.

Remark 3.1. This scheme is first order consistent in $\tau$ if $\theta \neq \frac{1}{2}$ and second order if $\theta=\frac{1}{2}$.

For linear elastodynamics (without unilateral contact), it is also known to be unconditionally stable for $\theta \geq \frac{1}{2}$ and conditionally stable when $\theta<\frac{1}{2}$. It is fully explicit when $\theta=0$. It is dissipative when $\theta>\frac{1}{2}$ and conserves the energy when $\theta=\frac{1}{2}$. 
Remark 3.2. The initial condition $\ddot{\mathbf{u}}^{h, 0}$ is determined in fact through:

$$
\left\langle\rho \ddot{\mathbf{u}}_{0}^{h}, \mathbf{v}^{h}\right\rangle=L^{0}\left(\mathbf{v}^{h}\right)-A_{\Theta \gamma_{h}}\left(\mathbf{u}_{0}^{h}, \mathbf{v}^{h}\right)-\int_{\Gamma_{C}} \frac{1}{\gamma_{h}}\left[P_{\gamma_{h}}\left(\mathbf{u}_{0}^{h}\right)\right]_{+} P_{\Theta \gamma_{h}}\left(\mathbf{v}^{h}\right) \mathrm{d} \Gamma \quad \forall \mathbf{v}^{h} \in \mathbf{V}^{h} .
$$

Thus $\ddot{\mathbf{u}}_{0}^{h}$ is obtained from $\mathbf{u}_{0}^{h}$ by inversion of the mass matrix $\mathbf{M}^{h}$.

\subsubsection{A Newmark scheme}

We semi-discretize in time problem (2.6) using a Newmark scheme, which parameters are $\beta \in[0,1 / 2]$, $\gamma \in[0,1]$. For $n \geq 0$, the fully discretized problem reads:

$$
\left\{\begin{array}{l}
\text { Find } \mathbf{u}^{h, n+1}, \dot{\mathbf{u}}^{h, n+1}, \ddot{\mathbf{u}}^{h, n+1} \in \mathbf{V}^{h} \text { such that: } \\
\mathbf{u}^{h, n+1}=\mathbf{u}^{h, n}+\tau \dot{\mathbf{u}}^{h, n}+\frac{\tau^{2}}{2} \ddot{\mathbf{u}}^{h, n+2 \beta}, \\
\dot{\mathbf{u}}^{h, n+1}=\dot{\mathbf{u}}^{h, n}+\tau \ddot{\mathbf{u}}^{h, n+\gamma}, \\
\left\langle\rho \ddot{\mathbf{u}}^{h, n+1}, \mathbf{v}^{h}\right\rangle+A_{\Theta \gamma_{h}}\left(\mathbf{u}^{h, n+1}, \mathbf{v}^{h}\right)+\int_{\Gamma_{C}} \frac{1}{\gamma_{h}}\left[P_{\gamma_{h}}\left(\mathbf{u}^{h, n+1}\right)\right]_{+} P_{\Theta \gamma_{h}}\left(\mathbf{v}^{h}\right) \mathrm{d} \Gamma=L^{n+1}\left(\mathbf{v}^{h}\right), \\
\quad \forall \mathbf{v}^{h} \in \mathbf{V}^{h},
\end{array}\right.
$$

with initial conditions $\mathbf{u}^{h, 0}=\mathbf{u}_{0}^{h}, \dot{\mathbf{u}}^{h, 0}=\dot{\mathbf{u}}_{0}^{h}, \ddot{\mathbf{u}}^{h, 0}=\ddot{\mathbf{u}}_{0}^{h}$ (see Rem. 3.2).

Remark 3.3. This scheme is first order consistent in $\tau$ when $\gamma \neq \frac{1}{2}$, second order when $\gamma=\frac{1}{2}$ and $\beta \neq \frac{1}{12}$, and fourth order when $\gamma=\frac{1}{2}$ and $\beta=\frac{1}{12}$. When applied to linear elastodynamics (without unilateral contact), it is not stable when $\gamma<\frac{1}{2}$, unconditionally stable when $\gamma \geq \frac{1}{2}$ and $\frac{\gamma}{2} \leq \beta \leq \frac{1}{2}$, and conditionally stable when $\gamma \geq \frac{1}{2}$ and $0 \leq \beta \leq \frac{\gamma}{2}$.

\subsubsection{A new hybrid scheme}

We introduce a new time-marching scheme for problem (2.6). Inspired by the works of Gonzalez [24] and Hauret and Le Tallec [30], the idea is to propose an hybrid discretization of the Nitsche-based contact term: the linear part of problem (2.6) is treated with a conservative Crank-Nicolson scheme, whereas the non-linear part arising from contact is discretized with a linear combination of Crank-Nicolson and Midpoint schemes. This strategy is of interest since the resulting scheme is unconditionally stable in the symmetric case and still second order consistent in time, as it will be shown in a forthcoming work (see [15]).

For $n \geq 0$, the fully discretized problem reads:

$$
\left\{\begin{array}{l}
\text { Find } \mathbf{u}^{h, n+1}, \dot{\mathbf{u}}^{h, n+1}, \ddot{\mathbf{u}}^{h, n+1} \in \mathbf{V}^{h} \text { such that: } \\
\mathbf{u}^{h, n+1}=\mathbf{u}^{h, n}+\tau \dot{\mathbf{u}}^{h, n+\frac{1}{2}} \\
\dot{\mathbf{u}}^{h, n+1}=\dot{\mathbf{u}}^{h, n}+\tau \ddot{\mathbf{u}}^{h, n+\frac{1}{2}} \\
\left\langle\rho \ddot{\mathbf{u}}^{h, n+\frac{1}{2}}, \mathbf{v}^{h}\right\rangle+A_{\Theta \gamma_{h}}\left(\mathbf{u}^{h, n+\frac{1}{2}}, \mathbf{v}^{h}\right)+\int_{\Gamma_{C}} \frac{1}{\gamma_{h}} \Phi\left(\mathbf{u}^{h, n}, \mathbf{u}^{h, n+1}\right) P_{\Theta \gamma_{h}}\left(\mathbf{v}^{h}\right) \mathrm{d} \Gamma=L^{n+\frac{1}{2}}\left(\mathbf{v}^{h}\right), \\
\quad \forall \mathbf{v}^{h} \in \mathbf{V}^{h}
\end{array}\right.
$$

with the initial conditions $\mathbf{u}^{h, 0}=\mathbf{u}_{0}^{h}, \dot{\mathbf{u}}^{h, 0}=\dot{\mathbf{u}}_{0}^{h}, \ddot{\mathbf{u}}^{h, 0}=\ddot{\mathbf{u}}_{0}^{h}$ (see Rem. 3.2) and with the following expression for $\Phi\left(\mathbf{u}^{h, n}, \mathbf{u}^{h, n+1}\right)$ :

$$
\Phi\left(\mathbf{u}^{h, n}, \mathbf{u}^{h, n+1}\right):=H\left(P_{\gamma_{h}}\left(\mathbf{u}^{h, n}\right)\right)\left[P_{\gamma_{h}}\left(\mathbf{u}^{h, n+\frac{1}{2}}\right)\right]_{+}+H\left(-P_{\gamma_{h}}\left(\mathbf{u}^{h, n}\right)\right)\left[P_{\gamma_{h}}\left(\mathbf{u}^{h}\right)\right]_{+}^{n+\frac{1}{2}} .
$$

Remark that $\left[P_{\gamma_{h}}\left(\mathbf{u}^{h}\right)\right]_{+}^{n+\frac{1}{2}}=\frac{1}{2}\left(\left[P_{\gamma_{h}}\left(\mathbf{u}^{h, n}\right)\right]_{+}+\left[P_{\gamma_{h}}\left(\mathbf{u}^{h, n+1}\right)\right]_{+}\right)$represents the Crank-Nicolson part, whereas $\left[P_{\gamma_{h}}\left(\mathbf{u}^{h, n+\frac{1}{2}}\right)\right]_{+}=\left[\frac{1}{2}\left(P_{\gamma_{h}}\left(\mathbf{u}^{h, n}\right)+P_{\gamma_{h}}\left(\mathbf{u}^{h, n+1}\right)\right)\right]_{+}$stands for the Midpoint part. So, when $P_{\gamma_{h}}\left(\mathbf{u}^{h, n}\right)>0$, the Midpoint scheme is applied, and when $P_{\gamma_{h}}\left(\mathbf{u}^{h, n}\right)<0$, the Crank-Nicolson scheme is applied instead. When $P_{\gamma_{h}}\left(\mathbf{u}^{h, n}\right)=0$ both schemes coincide. 


\subsection{Well-posedness of the fully discrete formulations}

Except for their explicit variants, that are obtained for $\theta=0$ ( $\theta$-scheme) and $\beta=0$ (Newmark), all the fully discrete formulations involve solving a non-linear problem at each time-step $n$. We study here conditions on numerical parameters upon which this non-linear problem admits a unique solution. We can apply each time an analogous argument as in [14]. One interesting consequence, as it will be shown, is that reducing the time-step $\tau$ weakens the condition $\gamma_{0}$ small whenever $\Theta \neq-1$.

\subsubsection{Well-posedness of the $\theta$-scheme}

The well-posedness of the $\theta$-scheme (3.1) is stated below:

\section{Proposition 3.4.}

1. If $\theta=0$, existence and uniqueness of (3.1) always holds since the scheme is fully explicit.

2. Let $\theta>0$. If

$$
(1+\Theta)^{2} \gamma_{0} \leq C\left(1+\frac{\rho h^{2}}{\tau^{2} \theta^{2}}\right)
$$

where $C$ is a positive constant, then at each time-step n, problem (3.1) admits one unique solution.

Proof. When $\theta>0$, expressing $\ddot{\mathbf{u}}^{h, n+1}$ as a function of $\mathbf{u}^{h, n+1}, \ddot{\mathbf{u}}^{h, n}, \dot{\mathbf{u}}^{h, n}, \mathbf{u}^{h, n}$, the $\theta$-scheme (3.1) can be written as follows: for $n \geq 0$ :

$$
\left\{\begin{array}{l}
\text { Find } \mathbf{u}^{h, n+1}, \dot{\mathbf{u}}^{h, n+1}, \ddot{\mathbf{u}}^{h, n+1} \in \mathbf{V}^{h} \text { such that: } \\
\mathbf{u}^{h, n+1}=\mathbf{u}^{h, n}+\tau \dot{\mathbf{u}}^{h, n+\theta} \\
\dot{\mathbf{u}}^{h, n+1}=\dot{\mathbf{u}}^{h, n}+\tau \ddot{\mathbf{u}}^{h, n+\theta} \\
\left\langle\frac{\rho}{\tau^{2} \theta^{2}} \mathbf{u}^{h, n+1}, \mathbf{v}^{h}\right\rangle+A_{\Theta \gamma_{h}}\left(\mathbf{u}^{h, n+1}, \mathbf{v}^{h}\right)+\int_{\Gamma_{C}} \frac{1}{\gamma_{h}}\left[P_{\gamma_{h}}\left(\mathbf{u}^{h, n+1}\right)\right]_{+} P_{\Theta \gamma_{h}}\left(\mathbf{v}^{h}\right) \mathrm{d} \Gamma \\
=L^{n+1}\left(\mathbf{v}^{h}\right)+\left\langle\frac{\rho(1-\theta)}{\theta} \ddot{\mathbf{u}}^{h, n}+\frac{\rho}{\tau \theta^{2}} \dot{\mathbf{u}}^{h, n}+\frac{\rho}{\tau^{2} \theta^{2}} \mathbf{u}^{h, n}, \mathbf{v}^{h}\right\rangle, \quad \forall \mathbf{v}^{h} \in \mathbf{V}^{h} .
\end{array}\right.
$$

Using the Riesz representation theorem, we define a non-linear operator $\mathbf{B}_{\theta}^{h}: \mathbf{V}^{h} \rightarrow \mathbf{V}^{h}$, by means of the formula:

$$
\left(\mathbf{B}_{\theta}^{h} \mathbf{v}^{h}, \mathbf{w}^{h}\right)_{1, \Omega}:=\left\langle\frac{\rho}{\tau^{2} \theta^{2}} \mathbf{v}^{h}, \mathbf{w}^{h}\right\rangle+A_{\Theta \gamma_{h}}\left(\mathbf{v}^{h}, \mathbf{w}^{h}\right)+\int_{\Gamma_{C}} \frac{1}{\gamma_{h}}\left[P_{\gamma_{h}}\left(\mathbf{v}^{h}\right)\right]_{+} P_{\Theta \gamma_{h}}\left(\mathbf{w}^{h}\right) \mathrm{d} \Gamma,
$$

for all $\mathbf{v}^{h}, \mathbf{w}^{h} \in \mathbf{V}^{h}$, and where $(\cdot, \cdot)_{1, \Omega}$ stands for the scalar product in $\left(H^{1}(\Omega)\right)^{d}$. Note that problem $(2.6)$ is well-posed if and only if $\mathbf{B}_{\theta}^{h}$ is a one-to-one operator. Let $\mathbf{v}^{h}, \mathbf{w}^{h} \in \mathbf{V}^{h}$. We have:

$$
\begin{aligned}
\left(\mathbf{B}_{\theta}^{h} \mathbf{v}^{h}-\mathbf{B}_{\theta}^{h} \mathbf{w}^{h}, \mathbf{v}^{h}-\mathbf{w}^{h}\right)_{1, \Omega}= & \left\langle\frac{\rho}{\tau^{2} \theta^{2}}\left(\mathbf{v}^{h}-\mathbf{w}^{h}\right), \mathbf{v}^{h}-\mathbf{w}^{h}\right\rangle+a\left(\mathbf{v}^{h}-\mathbf{w}^{h}, \mathbf{v}^{h}-\mathbf{w}^{h}\right)-\Theta\left\|\gamma_{h}^{\frac{1}{2}} \sigma_{n}\left(\mathbf{v}^{h}-\mathbf{w}^{h}\right)\right\|_{0, \Gamma_{C}}^{2} \\
& +\int_{\Gamma_{C}} \frac{1}{\gamma_{h}}\left(\left[P_{\gamma_{h}}\left(\mathbf{v}^{h}\right)\right]_{+}-\left[P_{\gamma_{h}}\left(\mathbf{w}^{h}\right)\right]_{+}\right)\left(v_{n}^{h}-w_{n}^{h}-\Theta \gamma_{h} \sigma_{n}\left(\mathbf{v}^{h}-\mathbf{w}^{h}\right)\right) \mathrm{d} \Gamma \\
= & \frac{\rho}{\tau^{2} \theta^{2}}\left\|\mathbf{v}^{h}-\mathbf{w}^{h}\right\|_{0, \Omega}^{2}+a\left(\mathbf{v}^{h}-\mathbf{w}^{h}, \mathbf{v}^{h}-\mathbf{w}^{h}\right)-\Theta\left\|\gamma_{h}^{\frac{1}{2}} \sigma_{n}\left(\mathbf{v}^{h}-\mathbf{w}^{h}\right)\right\|_{0, \Gamma_{C}}^{2} \\
& +\int_{\Gamma_{C}} \frac{1}{\gamma_{h}}\left(\left[P_{\gamma_{h}}\left(\mathbf{v}^{h}\right)\right]_{+}-\left[P_{\gamma_{h}}\left(\mathbf{w}^{h}\right)\right]_{+}\right) P_{\gamma_{h}}\left(\mathbf{v}^{h}-\mathbf{w}^{h}\right) \mathrm{d} \Gamma \\
& +(1-\Theta) \int_{\Gamma_{C}} \frac{1}{\gamma_{h}}\left(\left[P_{\gamma_{h}}\left(\mathbf{v}^{h}\right)\right]_{+}-\left[P_{\gamma_{h}}\left(\mathbf{w}^{h}\right)\right]_{+}\right) \gamma_{h} \sigma_{n}\left(\mathbf{v}^{h}-\mathbf{w}^{h}\right) \mathrm{d} \Gamma .
\end{aligned}
$$


Using the inequality (2.2) in (3.4), Cauchy-Schwarz inequality and the inverse inequality (2.8), we get

$$
\begin{aligned}
\left(\mathbf{B}_{\theta}^{h} \mathbf{v}^{h}-\mathbf{B}_{\theta}^{h} \mathbf{w}^{h}, \mathbf{v}^{h}-\mathbf{w}^{h}\right)_{1, \Omega} \geq & \frac{C \rho h^{2}}{\tau^{2} \theta^{2}}\left\|\mathbf{v}^{h}-\mathbf{w}^{h}\right\|_{1, \Omega}^{2}+a\left(\mathbf{v}^{h}-\mathbf{w}^{h}, \mathbf{v}^{h}-\mathbf{w}^{h}\right)-\Theta\left\|\gamma_{h}^{\frac{1}{2}} \sigma_{n}\left(\mathbf{v}^{h}-\mathbf{w}^{h}\right)\right\|_{0, \Gamma_{C}}^{2} \\
& +\left\|\gamma_{h} \frac{-1}{2}\left(\left[P_{\gamma_{h}}\left(\mathbf{v}^{h}\right)\right]_{+}-\left[P_{\gamma_{h}}\left(\mathbf{w}^{h}\right)\right]_{+}\right)\right\|_{0, \Gamma_{C}}^{2} \\
& -|1-\Theta|\left\|\gamma_{h}{ }^{-\frac{1}{2}}\left(\left[P_{\gamma_{h}}\left(\mathbf{v}^{h}\right)\right]_{+}-\left[P_{\gamma_{h}}\left(\mathbf{w}^{h}\right)\right]_{+}\right)\right\|_{0, \Gamma_{C}}\left\|\gamma_{h}^{\frac{1}{2}} \sigma_{n}\left(\mathbf{v}^{h}-\mathbf{w}^{h}\right)\right\|_{0, \Gamma_{C}} .
\end{aligned}
$$

If $\Theta=1$, we use the coercivity of $a(\cdot, \cdot)$ and the property (2.7) in the previous expression (3.5). Therefore there exists positive constants $C, C^{\prime}$ such that:

$$
\left(\mathbf{B}_{\theta}^{h} \mathbf{v}^{h}-\mathbf{B}_{\theta}^{h} \mathbf{w}^{h}, \mathbf{v}^{h}-\mathbf{w}^{h}\right)_{1, \Omega} \geq\left(C+\frac{C \rho h^{2}}{\tau^{2} \theta^{2}}-C^{\prime} \gamma_{0}\right)\left\|\mathbf{v}^{h}-\mathbf{w}^{h}\right\|_{1, \Omega}^{2} .
$$

We now suppose that $\Theta \neq 1$. Let $\beta>0$. Applying Young's inequality in (3.5) yields:

$$
\begin{aligned}
& \left(\mathbf{B}_{\theta}^{h} \mathbf{v}^{h}-\mathbf{B}_{\theta}^{h} \mathbf{w}^{h}, \mathbf{v}^{h}-\mathbf{w}^{h}\right)_{1, \Omega} \\
\geq & \frac{C \rho h^{2}}{\tau^{2} \theta^{2}}\left\|\mathbf{v}^{h}-\mathbf{w}^{h}\right\|_{1, \Omega}^{2}+a\left(\mathbf{v}^{h}-\mathbf{w}^{h}, \mathbf{v}^{h}-\mathbf{w}^{h}\right)-\Theta\left\|\gamma_{h}^{\frac{1}{2}} \sigma_{n}\left(\mathbf{v}^{h}-\mathbf{w}^{h}\right)\right\|_{0, \Gamma_{C}}^{2} \\
& +\left\|\gamma_{h}{ }^{-\frac{1}{2}}\left(\left[P_{\gamma_{h}}\left(\mathbf{v}^{h}\right)\right]_{+}-\left[P_{\gamma_{h}}\left(\mathbf{w}^{h}\right)\right]_{+}\right)\right\|_{0, \Gamma_{C}}^{2} \\
& -\frac{|1-\Theta|}{2 \beta}\left\|\gamma_{h}{ }^{-\frac{1}{2}}\left(\left[P_{\gamma_{h}}\left(\mathbf{v}^{h}\right)\right]_{+}-\left[P_{\gamma_{h}}\left(\mathbf{w}^{h}\right)\right]_{+}\right)\right\|_{0, \Gamma_{C}}^{2}-\frac{|1-\Theta| \beta}{2}\left\|\gamma_{h}^{\frac{1}{2}} \sigma_{n}\left(\mathbf{v}^{h}-\mathbf{w}^{h}\right)\right\|_{0, \Gamma_{C}}^{2} \\
= & \frac{C \rho h^{2}}{\tau^{2} \theta^{2}}\left\|\mathbf{v}^{h}-\mathbf{w}^{h}\right\|_{1, \Omega}^{2}+a\left(\mathbf{v}^{h}-\mathbf{w}^{h}, \mathbf{v}^{h}-\mathbf{w}^{h}\right)-\left(\Theta+\frac{|1-\Theta| \beta}{2}\right)\left\|\gamma_{h}^{\frac{1}{2}} \sigma_{n}\left(\mathbf{v}^{h}-\mathbf{w}^{h}\right)\right\|_{0, \Gamma_{C}}^{2} \\
& +\left(1-\frac{|1-\Theta|}{2 \beta}\right)\left\|\gamma_{h}{ }^{-\frac{1}{2}}\left(\left[P_{\gamma_{h}}\left(\mathbf{v}^{h}\right)\right]_{+}-\left[P_{\gamma_{h}}\left(\mathbf{w}^{h}\right)\right]_{+}\right)\right\|_{0, \Gamma_{C}}^{2} .
\end{aligned}
$$

Choosing $\beta=|1-\Theta| / 2$ in (3.7), we get:

$$
\begin{aligned}
& \left(\mathbf{B}_{\theta}^{h} \mathbf{v}^{h}-\mathbf{B}_{\theta}^{h} \mathbf{w}^{h}, \mathbf{v}^{h}-\mathbf{w}^{h}\right)_{1, \Omega} \\
\geq & \frac{C \rho h^{2}}{\tau^{2} \theta^{2}}\left\|\mathbf{v}^{h}-\mathbf{w}^{h}\right\|_{1, \Omega}^{2}+a\left(\mathbf{v}^{h}-\mathbf{w}^{h}, \mathbf{v}^{h}-\mathbf{w}^{h}\right)-\frac{1}{4}(1+\Theta)^{2}\left\|\gamma_{h}^{\frac{1}{2}} \sigma_{n}\left(\mathbf{v}^{h}-\mathbf{w}^{h}\right)\right\|_{0, \Gamma_{C}}^{2} \\
\geq & \left(C+\frac{C \rho h^{2}}{\tau^{2} \theta^{2}}-C^{\prime}(1+\Theta)^{2} \gamma_{0}\right)\left\|\mathbf{v}^{h}-\mathbf{w}^{h}\right\|_{1, \Omega}^{2},
\end{aligned}
$$

where $C, C^{\prime}$ are positive constants.

Next, let us show that $\mathbf{B}_{\theta}^{h}$ is also hemicontinuous. Since $\mathbf{V}^{h}$ is a vector space, it is sufficient to show that

$$
[0,1] \ni t \mapsto \varphi(t):=\left(\mathbf{B}_{\theta}^{h}\left(\mathbf{v}^{h}-t \mathbf{w}^{h}\right), \mathbf{w}^{h}\right)_{1, \Omega} \in \mathbb{R}
$$

is a continuous real function, for all $\mathbf{v}^{h}, \mathbf{w}^{h} \in \mathbf{V}^{h}$. Let $s, t \in[0,1]$, we have:

$$
\begin{aligned}
|\varphi(t)-\varphi(s)|= & \left|\left(\mathbf{B}_{\theta}^{h}\left(\mathbf{v}^{h}-t \mathbf{w}^{h}\right)-\mathbf{B}_{\theta}^{h}\left(\mathbf{v}^{h}-s \mathbf{w}^{h}\right), \mathbf{w}^{h}\right)_{1, \Omega}\right| \\
= & \mid(s-t)\left(\frac{\rho}{\tau^{2} \theta^{2}}\left\|\mathbf{w}^{h}\right\|_{0, \Omega}^{2}+A_{\Theta \gamma_{h}}\left(\mathbf{w}^{h}, \mathbf{w}^{h}\right)\right) \\
& +\int_{\Gamma_{C}} \frac{1}{\gamma_{h}}\left(\left[P_{\gamma_{h}}\left(\mathbf{v}^{h}-t \mathbf{w}^{h}\right)\right]_{+}-\left[P_{\gamma_{h}}\left(\mathbf{v}^{h}-s \mathbf{w}^{h}\right)\right]_{+}\right) P_{\Theta \gamma_{h}}\left(\mathbf{w}^{h}\right) \mathrm{d} \Gamma \mid \\
\leq & |s-t|\left(\frac{\rho}{\tau^{2} \theta^{2}}\left\|\mathbf{w}^{h}\right\|_{0, \Omega}^{2}+\left|A_{\Theta \gamma_{h}}\left(\mathbf{w}^{h}, \mathbf{w}^{h}\right)\right|\right) \\
& +\int_{\Gamma_{C}} \frac{1}{\gamma_{h}}\left|\left[P_{\gamma_{h}}\left(\mathbf{v}^{h}-t \mathbf{w}^{h}\right)\right]_{+}-\left[P_{\gamma_{h}}\left(\mathbf{v}^{h}-s \mathbf{w}^{h}\right)\right]_{+}\right|\left|P_{\Theta \gamma_{h}}\left(\mathbf{w}^{h}\right)\right| \mathrm{d} \Gamma .
\end{aligned}
$$


With help of the bound $\left|[a]_{+}-[b]_{+}\right| \leq|a-b|$, for all $a, b \in \mathbb{R}$, and using the linearity of $P_{\gamma_{h}}$, we deduce that:

$$
\begin{aligned}
& \int_{\Gamma_{C}} \frac{1}{\gamma_{h}}\left|\left[P_{\gamma_{h}}\left(\mathbf{v}^{h}-t \mathbf{w}^{h}\right)\right]_{+}-\left[P_{\gamma_{h}}\left(\mathbf{v}^{h}-s \mathbf{w}^{h}\right)\right]_{+}\right|\left|P_{\Theta \gamma_{h}}\left(\mathbf{w}^{h}\right)\right| \mathrm{d} \Gamma \\
\leq & \int_{\Gamma_{C}} \frac{1}{\gamma_{h}}\left|P_{\gamma_{h}}\left(\mathbf{v}^{h}-t \mathbf{w}^{h}\right)-P_{\gamma_{h}}\left(\mathbf{v}^{h}-s \mathbf{w}^{h}\right)\right|\left|P_{\Theta \gamma_{h}}\left(\mathbf{w}^{h}\right)\right| \mathrm{d} \Gamma \\
= & \int_{\Gamma_{C}} \frac{1}{\gamma_{h}}\left|(s-t) P_{\gamma_{h}}\left(\mathbf{w}^{h}\right)\right|\left|P_{\Theta \gamma_{h}}\left(\mathbf{w}^{h}\right)\right| \mathrm{d} \Gamma .
\end{aligned}
$$

It results that:

$$
|\varphi(t)-\varphi(s)| \leq|s-t|\left(\frac{\rho}{\tau^{2} \theta^{2}}\left\|\mathbf{w}^{h}\right\|_{0, \Omega}^{2}+\left|A_{\Theta \gamma_{h}}\left(\mathbf{w}^{h}, \mathbf{w}^{h}\right)\right|+\int_{\Gamma_{C}} \frac{1}{\gamma_{h}}\left|P_{\gamma_{h}}\left(\mathbf{w}^{h}\right)\right|\left|P_{\Theta \gamma_{h}}\left(\mathbf{w}^{h}\right)\right| \mathrm{d} \Gamma\right),
$$

which means that $\varphi$ is Lipschitz, so that $\mathbf{B}_{\theta}^{h}$ is hemicontinuous. Since properties (3.6) and (3.8) also hold, we finally apply the Corollary 15 (p. 126) of [9] to conclude that $\mathbf{B}_{\theta}^{h}$ is a one-to-one operator. This ends the proof when $\theta>0$.

When $\theta=0$, we simply carry out the following sequence of computations to go from time-step $n$ to time-step $n+1$ :

$$
\begin{aligned}
& \mathbf{u}^{h, n+1}=\mathbf{u}^{h, n}+\tau \dot{\mathbf{u}}^{h, n}, \\
& \dot{\mathbf{u}}^{h, n+1}=\dot{\mathbf{u}}^{h, n}+\tau \ddot{\mathbf{u}}^{h, n}, \\
& \left\langle\rho \ddot{\mathbf{u}}^{h, n+1}, \mathbf{v}^{h}\right\rangle=L^{n+1}\left(\mathbf{v}^{h}\right)-A_{\Theta \gamma_{h}}\left(\mathbf{u}^{h, n+1}, \mathbf{v}^{h}\right)-\int_{\Gamma_{C}} \frac{1}{\gamma_{h}}\left[P_{\gamma_{h}}\left(\mathbf{u}^{h, n+1}\right)\right]_{+} P_{\Theta \gamma_{h}}\left(\mathbf{v}^{h}\right) \mathrm{d} \Gamma, \\
& \forall \mathbf{v}^{h} \in \mathbf{V}^{h},
\end{aligned}
$$

and we note that the last step needs only the computation of $\left(\mathbf{M}^{h}\right)^{-1}$, or is explicit if a mass-lumping technique is used.

\subsubsection{Well-posedness of the Newmark scheme}

A similar result holds for the Newmark scheme (3.2). More precisely, we have:

\section{Proposition 3.5.}

1. If $\beta=0$, existence and uniqueness of (3.2) always holds since the scheme is fully explicit.

2. Let $\beta>0$. If

$$
(1+\Theta)^{2} \gamma_{0} \leq C\left(1+\frac{\rho h^{2}}{\tau^{2} \beta}\right)
$$

where $C$ is a positive constant, then at each time-step n, problem (3.2) admits one unique solution.

Proof. The proof is the same than for the $\theta$-scheme.

\subsubsection{Well-posedness of the hybrid scheme}

The well-posedness of the fully discrete scheme (3.3) is stated below.

Proposition 3.6. If the condition below is satisfied

$$
(1+\Theta)^{2} \gamma_{0} \leq C\left(1+\frac{\rho h^{2}}{\tau^{2}}\right)
$$

where $C$ is a positive constant, then at each time-step n, problem (3.3) admits one unique solution. 
Proof. Using the expression $\dot{\mathbf{u}}^{h, n+1}=\dot{\mathbf{u}}^{h, n}+\tau \ddot{\mathbf{u}}^{h, n+\frac{1}{2}}$ and then the relationship $\mathbf{u}^{h, n+1}=\mathbf{u}^{h, n}+\tau \dot{\mathbf{u}}^{h, n+\frac{1}{2}}$, we obtain that:

$$
\ddot{\mathbf{u}}^{h, n+\frac{1}{2}}=\frac{1}{\tau}\left(\dot{\mathbf{u}}^{h, n+1}-\dot{\mathbf{u}}^{h, n}\right)=\frac{2}{\tau^{2}} \mathbf{u}^{h, n+1}-\frac{2}{\tau^{2}} \mathbf{u}^{h, n}-\frac{2}{\tau} \dot{\mathbf{u}}^{h, n}
$$

Then the scheme (3.3) can be written as follows, for $n \geq 0$ :

$$
\left\{\begin{array}{l}
\text { Find } \mathbf{u}^{h, n+1}, \dot{\mathbf{u}}^{h, n+1}, \ddot{\mathbf{u}}^{h, n+1} \in \mathbf{V}^{h} \text { such that: } \\
\mathbf{u}^{h, n+1}=\mathbf{u}^{h, n}+\tau \dot{\mathbf{u}}^{h, n+\frac{1}{2}} \\
\dot{\mathbf{u}}^{h, n+1}=\dot{\mathbf{u}}^{h, n}+\tau \ddot{\mathbf{u}}^{h, n+\frac{1}{2}} \\
\left\langle\frac{4 \rho}{\tau^{2}} \mathbf{u}^{h, n+1}, \mathbf{v}^{h}\right\rangle+A_{\Theta \gamma_{h}}\left(\mathbf{u}^{h, n+1}, \mathbf{v}^{h}\right)+\int_{\Gamma_{C}} \frac{2}{\gamma_{h}} \Phi\left(\mathbf{u}^{h, n}, \mathbf{u}^{h, n+1}\right) P_{\Theta \gamma_{h}}\left(\mathbf{v}^{h}\right) \mathrm{d} \Gamma \\
=2 L^{n+\frac{1}{2}}\left(\mathbf{v}^{h}\right)-A_{\Theta \gamma_{h}}\left(\mathbf{u}^{h, n}, \mathbf{v}^{h}\right)+\left\langle\frac{4 \rho}{\tau} \dot{\mathbf{u}}^{h, n}+\frac{4 \rho}{\tau^{2}} \mathbf{u}^{h, n}, \mathbf{v}^{h}\right\rangle, \quad \forall \mathbf{v}^{h} \in \mathbf{V}^{h} .
\end{array}\right.
$$

Using the Riesz representation theorem, we define a non-linear operator $\mathbf{B}_{H}^{h}: \mathbf{V}^{h} \rightarrow \mathbf{V}^{h}$, by means of the formula:

$$
\left(\mathbf{B}_{H}^{h} \mathbf{v}^{h}, \mathbf{w}^{h}\right)_{1, \Omega}:=\left\langle\frac{4 \rho}{\tau^{2}} \mathbf{v}^{h}, \mathbf{w}^{h}\right\rangle+A_{\Theta \gamma_{h}}\left(\mathbf{v}^{h}, \mathbf{w}^{h}\right)+\int_{\Gamma_{C}} \frac{2}{\gamma_{h}} \Phi\left(\mathbf{u}^{h, n}, \mathbf{v}^{h}\right) P_{\Theta \gamma_{h}}\left(\mathbf{w}^{h}\right) \mathrm{d} \Gamma,
$$

for all $\mathbf{v}^{h}, \mathbf{w}^{h} \in \mathbf{V}^{h}$, and where $(\cdot, \cdot)_{1, \Omega}$ stands for the scalar product in $\left(H^{1}(\Omega)\right)^{d}$. Note that problem (2.6) is well-posed if and only if $\mathbf{B}_{H}^{h}$ is a one-to-one operator. Let $\mathbf{v}^{h}, \mathbf{w}^{h} \in \mathbf{V}^{h}$, we have:

$$
\begin{aligned}
& \left(\mathbf{B}_{H}^{h} \mathbf{v}^{h}-\mathbf{B}_{H}^{h} \mathbf{w}^{h}, \mathbf{v}^{h}-\mathbf{w}^{h}\right)_{1, \Omega} \\
& =\left\langle\frac{4 \rho}{\tau^{2}}\left(\mathbf{v}^{h}-\mathbf{w}^{h}\right), \mathbf{v}^{h}-\mathbf{w}^{h}\right\rangle \\
& +a\left(\mathbf{v}^{h}-\mathbf{w}^{h}, \mathbf{v}^{h}-\mathbf{w}^{h}\right)-\Theta\left\|\gamma_{h}^{\frac{1}{2}} \sigma_{n}\left(\mathbf{v}^{h}-\mathbf{w}^{h}\right)\right\|_{0, \Gamma_{C}}^{2} \\
& +\int_{\Gamma_{C}} \frac{2}{\gamma_{h}}\left(\Phi\left(\mathbf{u}^{h, n}, \mathbf{v}^{h}\right)-\Phi\left(\mathbf{u}^{h, n}, \mathbf{w}^{h}\right)\right)\left(v_{n}^{h}-w_{n}^{h}-\Theta \gamma_{h} \sigma_{n}\left(\mathbf{v}^{h}-\mathbf{w}^{h}\right)\right) \mathrm{d} \Gamma \\
& =\frac{4 \rho}{\tau^{2}}\left\|\mathbf{v}^{h}-\mathbf{w}^{h}\right\|_{0, \Omega}^{2}+a\left(\mathbf{v}^{h}-\mathbf{w}^{h}, \mathbf{v}^{h}-\mathbf{w}^{h}\right)-\Theta\left\|\gamma_{h^{\frac{1}{2}}} \sigma_{n}\left(\mathbf{v}^{h}-\mathbf{w}^{h}\right)\right\|_{0, \Gamma_{C}}^{2} \\
& +\int_{\Gamma_{C}} \frac{2}{\gamma_{h}}\left(\Phi\left(\mathbf{u}^{h, n}, \mathbf{v}^{h}\right)-\Phi\left(\mathbf{u}^{h, n}, \mathbf{w}^{h}\right)\right) P_{\gamma_{h}}\left(\mathbf{v}^{h}-\mathbf{w}^{h}\right) \mathrm{d} \Gamma \\
& +(1-\Theta) \int_{\Gamma_{C}} \frac{2}{\gamma_{h}}\left(\Phi\left(\mathbf{u}^{h, n}, \mathbf{v}^{h}\right)-\Phi\left(\mathbf{u}^{h, n}, \mathbf{w}^{h}\right)\right) \gamma_{h} \sigma_{n}\left(\mathbf{v}^{h}-\mathbf{w}^{h}\right) \mathrm{d} \Gamma .
\end{aligned}
$$


We detail the expression of:

$$
\begin{aligned}
& \int_{\Gamma_{C}} \frac{2}{\gamma_{h}}\left(\Phi\left(\mathbf{u}^{h, n}, \mathbf{v}^{h}\right)-\Phi\left(\mathbf{u}^{h, n}, \mathbf{w}^{h}\right)\right) P_{\gamma_{h}}\left(\mathbf{v}^{h}-\mathbf{w}^{h}\right) \mathrm{d} \Gamma \\
= & \int_{\Gamma_{C}} \frac{2}{\gamma_{h}} H\left(P_{\gamma_{h}}\left(\mathbf{u}^{h, n}\right)\right)\left(\left[P_{\gamma_{h}}\left(\frac{1}{2}\left(\mathbf{u}^{h, n}+\mathbf{v}^{h}\right)\right)\right]_{+}-\left[P_{\gamma_{h}}\left(\frac{1}{2}\left(\mathbf{u}^{h, n}+\mathbf{w}^{h}\right)\right)\right]_{+}\right) P_{\gamma_{h}}\left(\mathbf{v}^{h}-\mathbf{w}^{h}\right) \mathrm{d} \Gamma \\
& +\int_{\Gamma_{C}} \frac{2}{\gamma_{h}} H\left(-P_{\gamma_{h}}\left(\mathbf{u}^{h, n}\right)\right)\left(\frac{1}{2}\left(\left[P_{\gamma_{h}}\left(\mathbf{u}^{h, n}\right)\right]_{+}+\left[P_{\gamma_{h}}\left(\mathbf{v}^{h}\right)\right]_{+}\right)\right. \\
& \left.-\frac{1}{2}\left(\left[P_{\gamma_{h}}\left(\mathbf{u}^{h, n}\right)\right]_{+}+\left[P_{\gamma_{h}}\left(\mathbf{w}^{h}\right)\right]_{+}\right)\right) P_{\gamma_{h}}\left(\mathbf{v}^{h}-\mathbf{w}^{h}\right) \mathrm{d} \Gamma \\
= & \int_{\Gamma_{C}} \frac{1}{\gamma_{h}} H\left(P_{\gamma_{h}}\left(\mathbf{u}^{h, n}\right)\right)\left(\left[P_{\gamma_{h}}\left(\mathbf{u}^{h, n}+\mathbf{v}^{h}\right)\right]_{+}-\left[P_{\gamma_{h}}\left(\mathbf{u}^{h, n}+\mathbf{w}^{h}\right)\right]_{+}\right) P_{\gamma_{h}}\left(\left(\mathbf{u}^{h, n}+\mathbf{v}^{h}\right)-\left(\mathbf{u}^{h, n}+\mathbf{w}^{h}\right)\right) \mathrm{d} \Gamma \\
& +\int_{\Gamma_{C}} \frac{1}{\gamma_{h}} H\left(-P_{\gamma_{h}}\left(\mathbf{u}^{h, n}\right)\right)\left(\left[P_{\gamma_{h}}\left(\mathbf{v}^{h}\right)\right]_{+}-\left[P_{\gamma_{h}}\left(\mathbf{w}^{h}\right)\right]_{+}\right) P_{\gamma_{h}}\left(\mathbf{v}^{h}-\mathbf{w}^{h}\right) \mathrm{d} \Gamma
\end{aligned}
$$

Then with help of the inequality (2.2) we bound:

$$
\begin{aligned}
& \int_{\Gamma_{C}} \frac{2}{\gamma_{h}}\left(\Phi\left(\mathbf{u}^{h, n}, \mathbf{v}^{h}\right)-\Phi\left(\mathbf{u}^{h, n}, \mathbf{w}^{h}\right)\right) P_{\gamma_{h}}\left(\mathbf{v}^{h}-\mathbf{w}^{h}\right) \mathrm{d} \Gamma \\
\geq & \left\|H^{\frac{1}{2}}\left(P_{\gamma_{h}}\left(\mathbf{u}^{h, n}\right)\right) \gamma_{h}{ }^{-\frac{1}{2}}\left(\left[P_{\gamma_{h}}\left(\mathbf{u}^{h, n}+\mathbf{v}^{h}\right)\right]_{+}-\left[P_{\gamma_{h}}\left(\mathbf{u}^{h, n}+\mathbf{w}^{h}\right)\right]_{+}\right)\right\|_{0, \Gamma_{C}}^{2} \\
& +\left\|H^{\frac{1}{2}}\left(-P_{\gamma_{h}}\left(\mathbf{u}^{h, n}\right)\right) \gamma_{h}{ }^{-\frac{1}{2}}\left(\left[P_{\gamma_{h}}\left(\mathbf{v}^{h}\right)\right]_{+}-\left[P_{\gamma_{h}}\left(\mathbf{w}^{h}\right)\right]_{+}\right)\right\|_{0, \Gamma_{C}}^{2} .
\end{aligned}
$$

With Cauchy-Schwarz and Young's inequalities, we also bound:

$$
\begin{aligned}
& (1-\Theta) \int_{\Gamma_{C}} \frac{2}{\gamma_{h}}\left(\Phi\left(\mathbf{u}^{h, n}, \mathbf{v}^{h}\right)-\Phi\left(\mathbf{u}^{h, n}, \mathbf{w}^{h}\right)\right) \gamma_{h} \sigma_{n}\left(\mathbf{v}^{h}-\mathbf{w}^{h}\right) \mathrm{d} \Gamma \\
= & (1-\Theta) \int_{\Gamma_{C}} \frac{1}{\gamma_{h}} H\left(P_{\gamma_{h}}\left(\mathbf{u}^{h, n}\right)\right)\left(\left[P_{\gamma_{h}}\left(\mathbf{u}^{h, n}+\mathbf{v}^{h}\right)\right]_{+}-\left[P_{\gamma_{h}}\left(\mathbf{u}^{h, n}+\mathbf{w}^{h}\right)\right]_{+}\right) \gamma_{h} \sigma_{n}\left(\mathbf{v}^{h}-\mathbf{w}^{h}\right) \mathrm{d} \Gamma \\
& +(1-\Theta) \int_{\Gamma_{C}} \frac{1}{\gamma_{h}} H\left(-P_{\gamma_{h}}\left(\mathbf{u}^{h, n}\right)\right)\left(\left[P_{\gamma_{h}}\left(\mathbf{v}^{h}\right)\right]_{+}-\left[P_{\gamma_{h}}\left(\mathbf{w}^{h}\right)\right]_{+}\right) \gamma_{h} \sigma_{n}\left(\mathbf{v}^{h}-\mathbf{w}^{h}\right) \mathrm{d} \Gamma \\
\leq & |1-\Theta|\left[\frac { 1 } { 2 \beta } \left(\left\|H^{\frac{1}{2}}\left(P_{\gamma_{h}}\left(\mathbf{u}^{h, n}\right)\right) \gamma_{h}^{-\frac{1}{2}}\left(\left[P_{\gamma_{h}}\left(\mathbf{u}^{h, n}+\mathbf{v}^{h}\right)\right]_{+}-\left[P_{\gamma_{h}}\left(\mathbf{u}^{h, n}+\mathbf{w}^{h}\right)\right]_{+}\right)\right\|_{0, \Gamma_{C}}^{2}\right.\right. \\
& \left.+\left\|H^{\frac{1}{2}}\left(-P_{\gamma_{h}}\left(\mathbf{u}^{h, n}\right)\right) \gamma_{h}^{-\frac{1}{2}}\left(\left[P_{\gamma_{h}}\left(\mathbf{v}^{h}\right)\right]_{+}-\left[P_{\gamma_{h}}\left(\mathbf{w}^{h}\right)\right]_{+}\right)\right\|_{0, \Gamma_{C}}^{2}\right) \\
& \left.+\frac{\beta}{2}\left(\left\|H^{\frac{1}{2}}\left(P_{\gamma_{h}}\left(\mathbf{u}^{h, n}\right)\right) \gamma_{h}^{\frac{1}{2}} \sigma_{n}\left(\mathbf{v}^{h}-\mathbf{w}^{h}\right)\right\|_{0, \Gamma_{C}}^{2}+\left\|H^{\frac{1}{2}}\left(-P_{\gamma_{h}}\left(\mathbf{u}^{h, n}\right)\right) \gamma_{h}^{\frac{1}{2}} \sigma_{n}\left(\mathbf{v}^{h}-\mathbf{w}^{h}\right)\right\|_{0, \Gamma_{C}}^{2}\right)\right],
\end{aligned}
$$

with $\beta>0$. With the property (2.3) we get:

$$
\begin{aligned}
& \quad(1-\Theta) \int_{\Gamma_{C}} \frac{2}{\gamma_{h}}\left(\Phi\left(\mathbf{u}^{h, n}, \mathbf{v}^{h}\right)-\Phi\left(\mathbf{u}^{h, n}, \mathbf{w}^{h}\right)\right) \gamma_{h} \sigma_{n}\left(\mathbf{v}^{h}-\mathbf{w}^{h}\right) \mathrm{d} \Gamma \\
& \leq|1-\Theta|\left[\frac { 1 } { 2 \beta } \left(\left\|H^{\frac{1}{2}}\left(P_{\gamma_{h}}\left(\mathbf{u}^{h, n}\right)\right) \gamma_{h}{ }^{-\frac{1}{2}}\left(\left[P_{\gamma_{h}}\left(\mathbf{u}^{h, n}+\mathbf{v}^{h}\right)\right]_{+}-\left[P_{\gamma_{h}}\left(\mathbf{u}^{h, n}+\mathbf{w}^{h}\right)\right]_{+}\right)\right\|_{0, \Gamma_{C}}^{2}\right.\right. \\
& \left.\left.\quad+\left\|H^{\frac{1}{2}}\left(-P_{\gamma_{h}}\left(\mathbf{u}^{h, n}\right)\right) \gamma_{h}{ }^{-\frac{1}{2}}\left(\left[P_{\gamma_{h}}\left(\mathbf{v}^{h}\right)\right]_{+}-\left[P_{\gamma_{h}}\left(\mathbf{w}^{h}\right)\right]_{+}\right)\right\|_{0, \Gamma_{C}}^{2}\right)+\frac{\beta}{2}\left\|\gamma_{h}^{\frac{1}{2}} \sigma_{n}\left(\mathbf{v}^{h}-\mathbf{w}^{h}\right)\right\|_{0, \Gamma_{C}}^{2}\right] .
\end{aligned}
$$


Using (3.10) and (3.11) in (3.9) and the inverse inequality (2.8), we obtain:

$$
\begin{aligned}
& \left(\mathbf{B}_{H}^{h} \mathbf{v}^{h}-\mathbf{B}_{H}^{h} \mathbf{w}^{h}, \mathbf{v}^{h}-\mathbf{w}^{h}\right)_{1, \Omega} \\
\geq & \frac{4 C \rho h^{2}}{\tau^{2}}\left\|\mathbf{v}^{h}-\mathbf{w}^{h}\right\|_{1, \Omega}^{2}+a\left(\mathbf{v}^{h}-\mathbf{w}^{h}, \mathbf{v}^{h}-\mathbf{w}^{h}\right)-\left(\Theta+|1-\Theta| \frac{\beta}{2}\right)\left\|\gamma_{h}^{\frac{1}{2}} \sigma_{n}\left(\mathbf{v}^{h}-\mathbf{w}^{h}\right)\right\|_{0, \Gamma_{C}}^{2} \\
& +\left(1-\frac{|1-\Theta|}{2 \beta}\right)\left(\left\|H^{\frac{1}{2}}\left(P_{\gamma_{h}}\left(\mathbf{u}^{h, n}\right)\right) \gamma_{h}-\frac{1}{2}\left(\left[P_{\gamma_{h}}\left(\mathbf{u}^{h, n}+\mathbf{v}^{h}\right)\right]_{+}-\left[P_{\gamma_{h}}\left(\mathbf{u}^{h, n}+\mathbf{w}^{h}\right)\right]_{+}\right)\right\|_{0, \Gamma_{C}}^{2}\right. \\
& \left.+\left\|H^{\frac{1}{2}}\left(-P_{\gamma_{h}}\left(\mathbf{u}^{h, n}\right)\right) \gamma_{h}{ }^{-\frac{1}{2}}\left(\left[P_{\gamma_{h}}\left(\mathbf{v}^{h}\right)\right]_{+}-\left[P_{\gamma_{h}}\left(\mathbf{w}^{h}\right)\right]_{+}\right)\right\|_{0, \Gamma_{C}}^{2}\right) .
\end{aligned}
$$

If $\Theta=1$, we use the coercivity of $a(\cdot, \cdot)$ and the property (2.7) in the previous expression (3.12). Therefore there exists positive constants $C, C^{\prime}$ such that:

$$
\left(\mathbf{B}_{H}^{h} \mathbf{v}^{h}-\mathbf{B}_{H}^{h} \mathbf{w}^{h}, \mathbf{v}^{h}-\mathbf{w}^{h}\right)_{1, \Omega} \geq\left(C+\frac{4 C \rho h^{2}}{\tau^{2}}-C^{\prime} \gamma_{0}\right)\left\|\mathbf{v}^{h}-\mathbf{w}^{h}\right\|_{1, \Omega}^{2}
$$

We now suppose that $\Theta \neq 1$. Choosing $\beta=|1-\Theta| / 2$ in (3.12), we get:

$$
\begin{aligned}
& \left(\mathbf{B}_{H}^{h} \mathbf{v}^{h}-\mathbf{B}_{H}^{h} \mathbf{w}^{h}, \mathbf{v}^{h}-\mathbf{w}^{h}\right)_{1, \Omega} \\
\geq & \frac{4 C \rho h^{2}}{\tau^{2}}\left\|\mathbf{v}^{h}-\mathbf{w}^{h}\right\|_{1, \Omega}^{2}+a\left(\mathbf{v}^{h}-\mathbf{w}^{h}, \mathbf{v}^{h}-\mathbf{w}^{h}\right)-\frac{1}{4}(1+\Theta)^{2}\left\|\gamma_{h}^{\frac{1}{2}} \sigma_{n}\left(\mathbf{v}^{h}-\mathbf{w}^{h}\right)\right\|_{0, \Gamma_{C}}^{2} \\
\geq & \left(C+\frac{4 C \rho h^{2}}{\tau^{2}}-C^{\prime}(1+\Theta)^{2} \gamma_{0}\right)\left\|\mathbf{v}^{h}-\mathbf{w}^{h}\right\|_{1, \Omega}^{2},
\end{aligned}
$$

where $C, C^{\prime}$ are positive constants.

Next, let us show that $\mathbf{B}_{H}^{h}$ is also hemicontinuous. Since $\mathbf{V}^{h}$ is a vector space, it is sufficient to show that

$$
[0,1] \ni t \mapsto \varphi(t):=\left(\mathbf{B}_{H}^{h}\left(\mathbf{v}^{h}-t \mathbf{w}^{h}\right), \mathbf{w}^{h}\right)_{1, \Omega} \in \mathbb{R}
$$

is a continuous real function, for all $\mathbf{v}^{h}, \mathbf{w}^{h} \in \mathbf{V}^{h}$. Let $s, t \in[0,1]$, we have:

$$
\begin{aligned}
|\varphi(t)-\varphi(s)|= & \left|\left(\mathbf{B}_{H}^{h}\left(\mathbf{v}^{h}-t \mathbf{w}^{h}\right)-\mathbf{B}_{H}^{h}\left(\mathbf{v}^{h}-s \mathbf{w}^{h}\right), \mathbf{w}^{h}\right)_{1, \Omega}\right| \\
= & \mid(s-t)\left(\frac{4 \rho}{\tau^{2}}\left\|\mathbf{w}^{h}\right\|_{0, \Omega}^{2}+A_{\Theta \gamma_{h}}\left(\mathbf{w}^{h}, \mathbf{w}^{h}\right)\right) \\
& +\int_{\Gamma_{C}} \frac{2}{\gamma_{h}}\left(\Phi\left(\mathbf{u}^{h, n}, \mathbf{v}^{h}-t \mathbf{w}^{h}\right)-\Phi\left(\mathbf{u}^{h, n}, \mathbf{v}^{h}-s \mathbf{w}^{h}\right)\right) P_{\Theta \gamma_{h}}\left(\mathbf{w}^{h}\right) \mathrm{d} \Gamma \mid \\
\leq & |s-t|\left(\frac{4 \rho}{\tau^{2}}\left\|\mathbf{w}^{h}\right\|_{0, \Omega}^{2}+\left|A_{\Theta \gamma_{h}}\left(\mathbf{w}^{h}, \mathbf{w}^{h}\right)\right|\right) \\
& +\int_{\Gamma_{C}} \frac{2}{\gamma_{h}}\left|\Phi\left(\mathbf{u}^{h, n}, \mathbf{v}^{h}-t \mathbf{w}^{h}\right)-\Phi\left(\mathbf{u}^{h, n}, \mathbf{v}^{h}-s \mathbf{w}^{h}\right)\right|\left|P_{\Theta \gamma_{h}}\left(\mathbf{w}^{h}\right)\right| \mathrm{d} \Gamma .
\end{aligned}
$$


With help of the bound $\left|[a]_{+}-[b]_{+}\right| \leq|a-b|$, for all $a, b \in \mathbb{R}$, using the linearity of $P_{\gamma_{h}}$ and (2.3) we deduce that:

$$
\begin{aligned}
& \int_{\Gamma_{C}} \frac{2}{\gamma_{h}}\left|\Phi\left(\mathbf{u}^{h, n}, \mathbf{v}^{h}-t \mathbf{w}^{h}\right)-\Phi\left(\mathbf{u}^{h, n}, \mathbf{v}^{h}-s \mathbf{w}^{h}\right)\right|\left|P_{\Theta \gamma_{h}}\left(\mathbf{w}^{h}\right)\right| \mathrm{d} \Gamma \\
\leq & \int_{\Gamma_{C}} \frac{2}{\gamma_{h}} H\left(P_{\gamma_{h}}\left(\mathbf{u}^{h, n}\right)\right)\left|\frac{1}{2}\left(\left[P_{\gamma_{h}}\left(\mathbf{u}^{h, n}+\mathbf{v}^{h}-t \mathbf{w}^{h}\right)\right]_{+}-\left[P_{\gamma_{h}}\left(\mathbf{u}^{h, n}+\mathbf{v}^{h}-s \mathbf{w}^{h}\right)\right]_{+}\right)\right|\left|P_{\Theta \gamma_{h}}\left(\mathbf{w}^{h}\right)\right| \mathrm{d} \Gamma \\
& +\int_{\Gamma_{C}} \frac{2}{\gamma_{h}} H\left(-P_{\gamma_{h}}\left(\mathbf{u}^{h, n}\right)\right)\left|\frac{1}{2}\left(\left[P_{\gamma_{h}}\left(\mathbf{v}^{h}-t \mathbf{w}^{h}\right)\right]_{+}-\left[P_{\gamma_{h}}\left(\mathbf{v}^{h}-s \mathbf{w}^{h}\right)\right]_{+}\right)\right|\left|P_{\Theta \gamma_{h}}\left(\mathbf{w}^{h}\right)\right| \mathrm{d} \Gamma \\
\leq & \int_{\Gamma_{C}} \frac{1}{\gamma_{h}} H\left(P_{\gamma_{h}}\left(\mathbf{u}^{h, n}\right)\right)\left|P_{\gamma_{h}}\left(\mathbf{u}^{h, n}+\mathbf{v}^{h}-t \mathbf{w}^{h}\right)-P_{\gamma_{h}}\left(\mathbf{u}^{h, n}+\mathbf{v}^{h}-s \mathbf{w}^{h}\right)\right|\left|P_{\Theta \gamma_{h}}\left(\mathbf{w}^{h}\right)\right| \mathrm{d} \Gamma \\
& +\int_{\Gamma_{C}} \frac{1}{\gamma_{h}} H\left(-P_{\gamma_{h}}\left(\mathbf{u}^{h, n}\right)\right)\left|P_{\gamma_{h}}\left(\mathbf{v}^{h}-t \mathbf{w}^{h}\right)-P_{\gamma_{h}}\left(\mathbf{v}^{h}-s \mathbf{w}^{h}\right)\right|\left|P_{\Theta \gamma_{h}}\left(\mathbf{w}^{h}\right)\right| \mathrm{d} \Gamma \\
= & \int_{\Gamma_{C}} \frac{1}{\gamma_{h}}\left|P_{\gamma_{h}}\left(\mathbf{v}^{h}-t \mathbf{w}^{h}\right)-P_{\gamma_{h}}\left(\mathbf{v}^{h}-s \mathbf{w}^{h}\right)\right|\left|P_{\Theta \gamma_{h}}\left(\mathbf{w}^{h}\right)\right| \mathrm{d} \Gamma \\
= & \int_{\Gamma_{C}} \frac{1}{\gamma_{h}}\left|(s-t) P_{\gamma_{h}}\left(\mathbf{w}^{h}\right)\right|\left|P_{\Theta \gamma_{h}}\left(\mathbf{w}^{h}\right)\right| \mathrm{d} \Gamma .
\end{aligned}
$$

It results that:

$$
|\varphi(t)-\varphi(s)| \leq|s-t|\left(\frac{4 \rho}{\tau^{2}}\left\|\mathbf{w}^{h}\right\|_{0, \Omega}^{2}+\left|A_{\Theta \gamma_{h}}\left(\mathbf{w}^{h}, \mathbf{w}^{h}\right)\right|+\int_{\Gamma_{C}} \frac{1}{\gamma_{h}}\left|P_{\gamma_{h}}\left(\mathbf{w}^{h}\right)\right|\left|P_{\Theta \gamma_{h}}\left(\mathbf{w}^{h}\right)\right| \mathrm{d} \Gamma\right),
$$

which means that $\varphi$ is Lipschitz, so that $\mathbf{B}_{H}^{h}$ is hemicontinuous. Since properties (3.13) and (3.14) also hold, we finally apply the Corollary 15 (p. 126) of [9] to conclude that $\mathbf{B}_{H}^{h}$ is a one-to-one operator. This ends the proof.

\section{Conclusion And Perspectives}

In this paper dealing with frictionless unilateral contact in the small displacements and deformations framework, we extend to the elastodynamic case the Nitsche-based method previously defined and analyzed in the elastostatic case. The resulting space (semi-)discretization is shown to be well-posed and we derive some stability results. For the symmetric variant of Nitsche's method, we prove the conservation of an augmented energy. We then define several time-marching schemes for which we obtain appropriate CFL conditions ensuring wellposedness.

The stability of the time-marching schemes and the corresponding numerical experiments can be found in reference [15].

\section{Appendix A. Estimate For THE INVERSE of THE DiscRETE MASS OPERATOR}

Lemma A.1. Suppose that the mesh $\mathcal{T}^{h}$ is quasi-uniform. Then there exists $C>0$ independent of $\rho, \gamma_{0}$ and $h$ such that:

$$
\left\|\left(\mathbf{M}^{h}\right)^{-1}\right\|_{\gamma_{h}} \leq C \rho^{-1}\left(1+\gamma_{0}^{-1}\right) h^{-2}
$$

where $\|\cdot\|_{\gamma_{h}}$ is the operator norm induced by the vector norm $\|\cdot\|_{\gamma_{h}}$ in $\mathbf{V}^{h}$.

Proof. We start from the definition of the operator norm, then use the (obvious) invertibility of $\mathbf{M}^{h}$ :

$$
\left\|\left(\mathbf{M}^{h}\right)^{-1}\right\|_{\gamma_{h}}=\sup _{\mathbf{v}^{h} \in \mathbf{V}^{h}} \frac{\left\|\left(\mathbf{M}^{h}\right)^{-1} \mathbf{v}^{h}\right\|_{\gamma_{h}}}{\left\|\mathbf{v}^{h}\right\|_{\gamma_{h}}}=\sup _{\mathbf{v}^{h} \in \mathbf{V}^{h}} \frac{\left\|\mathbf{v}^{h}\right\|_{\gamma_{h}}}{\left\|\mathbf{M}^{h} \mathbf{v}^{h}\right\|_{\gamma_{h}}} .
$$


Let $\mathbf{v}^{h} \in \mathbf{V}^{h}$. We now bound from below the norm $\left\|\mathbf{M}^{h} \mathbf{v}^{h}\right\|_{\gamma_{h}}$, with first:

$$
\left\|\mathbf{M}^{h} \mathbf{v}^{h}\right\|_{\gamma_{h}}=\sup _{\mathbf{w}^{h} \in \mathbf{V}^{h}} \frac{\left(\mathbf{M}^{h} \mathbf{v}^{h}, \mathbf{w}^{h}\right)_{\gamma_{h}}}{\left\|\mathbf{w}^{h}\right\|_{\gamma_{h}}}=\sup _{\mathbf{w}^{h} \in \mathbf{V}^{h}} \frac{\left\langle\rho \mathbf{v}^{h}, \mathbf{w}^{h}\right\rangle}{\left\|\mathbf{w}^{h}\right\|_{\gamma_{h}}} \geq \rho \frac{\left\langle\mathbf{v}^{h}, \mathbf{v}^{h}\right\rangle}{\left\|\mathbf{v}^{h}\right\|_{\gamma_{h}}}=\rho \frac{\left\|\mathbf{v}^{h}\right\|_{0, \Omega}^{2}}{\left\|\mathbf{v}^{h}\right\|_{\gamma_{h}}} .
$$

From ([8], Thm. 1.6.6) the following trace inequality holds:

$$
\left\|v_{n}^{h}\right\|_{0, \Gamma_{C}}^{2} \leq C\left\|\mathbf{v}^{h}\right\|_{0, \Omega}\left\|\mathbf{v}^{h}\right\|_{1, \Omega} .
$$

Then we use the quasi-uniformity of the mesh $\mathcal{T}_{h}$, the trace inequality (A.4) and two times the inverse inequality (2.8) to obtain:

$$
\begin{aligned}
\left\|\mathbf{v}^{h}\right\|_{\gamma_{h}}^{2} & =\left\|\mathbf{v}^{h}\right\|_{1, \Omega}^{2}+\left\|\gamma_{h}{ }^{-\frac{1}{2}} v_{n}^{h}\right\|_{0, \Gamma_{C}}^{2} \\
& \leq\left\|\mathbf{v}^{h}\right\|_{1, \Omega}^{2}+C\left(\gamma_{0} h\right)^{-1}\left\|v_{n}^{h}\right\|_{0, \Gamma_{C}}^{2} \\
& \leq C h^{-2}\left\|\mathbf{v}^{h}\right\|_{0, \Omega}^{2}+C\left(\gamma_{0} h\right)^{-1}\left\|\mathbf{v}^{h}\right\|_{0, \Omega}\left\|\mathbf{v}^{h}\right\|_{1, \Omega} \\
& \leq C h^{-2}\left\|\mathbf{v}^{h}\right\|_{0, \Omega}^{2}+C\left(\gamma_{0}\right)^{-1} h^{-2}\left\|\mathbf{v}^{h}\right\|_{0, \Omega}^{2} \\
& \leq C\left(1+\gamma_{0}^{-1}\right) h^{-2}\left\|\mathbf{v}^{h}\right\|_{0, \Omega}^{2} .
\end{aligned}
$$

We reinject this estimate into (A.3) to end up with bounding from below $\left\|\mathbf{M}^{h} \mathbf{v}^{h}\right\|_{\gamma_{h}}$ :

$$
\left\|\mathbf{M}^{h} \mathbf{v}^{h}\right\|_{\gamma_{h}} \geq C \rho \frac{h^{2}}{1+\gamma_{0}^{-1}}\left\|\mathbf{v}^{h}\right\|_{\gamma_{h}}
$$

This last inequality can be rewritten as

$$
\frac{\left\|\mathbf{v}^{h}\right\|_{\gamma_{h}}}{\left\|\mathbf{M}^{h} \mathbf{v}^{h}\right\|_{\gamma_{h}}} \leq C \rho^{-1}\left(1+\gamma_{0}^{-1}\right) h^{-2}
$$

Combined with (A.2), this ends the proof.

Acknowledgements. We thank the anonymous referees for their comments that helped to improve the paper.

\section{REFERENCES}

[1] R.A. Adams, Sobolev spaces. Pure Appl. Math., vol. 65. Academic Press, New York, London (1975).

[2] J. Ahn and D.E. Stewart, Existence of solutions for a class of impact problems without viscosity. SIAM J. Math. Anal. 38 (2006) 37-63.

[3] P. Alart and A. Curnier, A generalized newton method for contact problems with friction. J. Mech. Theor. Appl. 7 (1988) 67-82.

[4] F. Armero and E. Petőcz, Formulation and analysis of conserving algorithms for frictionless dynamic contact/impact problems. Comput. Methods Appl. Mech. Eng. 158 (1998) 269-300.

[5] M. Astorino, F. Chouly, and M.A. Fernández, An added-mass free semi-implicit coupling scheme for fluid-structure interaction. C. R. Math. Acad. Sci. Paris 347 (2009) 99-104.

[6] J.-P. Aubin and A. Cellina, Differential inclusions, vol. 264 of Grundlehren der Mathematischen Wissenschaften. SpringerVerlag, Berlin (1984).

[7] R. Becker, P. Hansbo, and R. Stenberg, A finite element method for domain decomposition with non-matching grids. ESAIM: M2AN 37 (2003) 209-225.

[8] S.-C. Brenner and L.-R. Scott, The Mathematical Theory of Finite Element Methods, vol. 15 of Texts Appl. Math. SpringerVerlag, New York, 2007.

[9] H. Brezis, Équations et inéquations non linéaires dans les espaces vectoriels en dualité. Ann. Inst. Fourier 18 (1968) $115-175$.

[10] E. Burman and M.A. Fernández, Stabilization of explicit coupling in fluid-structure interaction involving fluid incompressibility. Comput. Methods Appl. Mech. Eng. 198 (2009) 766-784. 
[11] G. Choudury and I. Lasiecka, Optimal convergence rates for semidiscrete approximations of parabolic problems with nonsmooth boundary data. Numer. Funct. Anal. Optim. 12 (1991) 469-485 (1992).

[12] F. Chouly, An adaptation of Nitsche's method to the Tresca friction problem. J. Math. Anal. Appl. 411 (2014) $329-339$.

[13] F. Chouly and P. Hild, A Nitsche-based method for unilateral contact problems: numerical analysis. SIAM J. Numer. Anal. 51 (2013) 1295-1307.

[14] F. Chouly, P. Hild, and Y. Renard, Symmetric and non-symmetric variants of Nitsche's method for contact problems in elasticity: theory and numerical experiments. Math. Comp. (2014). DOI:10.1090/S0025-5718-02913-X.

[15] F. Chouly, P. Hild and Y. Renard, A Nitsche finite element method for dynamic contact. 2. Stability of the schemes and numerical experiments. ESAIM: M2AN 49 (2015) 503-528.

[16] P.G. Ciarlet, Handbook of Numerical Analysis. The finite element method for elliptic problems. Edited by P.G. Ciarlet and J.L. Lions. In vol II, chap. 1. North Holland (1991) 17-352.

[17] F. Dabaghi, A. Petrov, J. Pousin, and Y. Renard, Convergence of mass redistribution method for the one-dimensional wave equation with a unilateral constraint at the boundary. ESAIM: M2AN 48 (2014) 1147-1169.

[18] C. D'Angelo and P. Zunino, Numerical approximation with Nitsche's coupling of transient Stokes'/Darcy's flow problems applied to hemodynamics. Appl. Numer. Math. 62 (2012) 378-395.

[19] R. Dautray and J.-L. Lions, Analyse mathématique et calcul numérique pour les sciences et les techniques. Évolution: semigroupe, variationnel. Vol. 8. Masson, Paris (1988).

[20] K. Deimling, Multivalued differential equations. In vol. 1 of de Gruyter Series Nonlin. Anal. Appl. Walter de Gruyter \& Co., Berlin (1992).

[21] C. Eck, J. Jarušek, and M. Krbec, Unilateral contact problems. In vol. 270 of Pure Appl. Math. Chapman \& Hall/CRC, Boca Raton, FL (2005).

[22] A. Ern and J.-L. Guermond, Theory and practice of finite elements. In vol. 159 of Appl. Math. Sci. Springer-Verlag, New York (2004).

[23] R. Glowinski and P. Le Tallec, Augmented Lagrangian and operator-splitting methods in nonlinear mechanics. In vol. 9 of SIAM Studies Appl. Math. Society for Industrial and Applied Mathematics, Philadelphia, PA (1989).

[24] O. Gonzalez, Exact energy and momentum conserving algorithms for general models in nonlinear elasticity. Comput. Methods Appl. Mech. Eng. 190 (2000) 1763-1783.

[25] W. Han and M. Sofonea, Quasistatic contact problems in viscoelasticity and viscoplasticity. In vol. 30 of AMS/IP Stud. Adv. Math. American Mathematical Society, Providence, RI (2002).

[26] A. Hansbo and P. Hansbo, A finite element method for the simulation of strong and weak discontinuities in solid mechanics. Comput. Methods Appl. Mech. Eng. 193 (2004) 3523-3540.

[27] P. Hansbo, Nitsche's method for interface problems in computational mechanics. GAMM-Mitt. 28 (2005) $183-206$.

[28] P. Hansbo, J. Hermansson, and T. Svedberg, Nitsche's method combined with space-time finite elements for ALE fluid-structure interaction problems. Comput. Methods Appl. Mech. Eng. 193 (2004) 4195-4206.

[29] J. Haslinger, I. Hlaváček, and J. Nečas, Handbook of Numerical Analysis. Numerical methods for unilateral problems in solid mechanics. Edited by P.G. Ciarlet and J.L. Lions. In Vol. IV, chap. 2. North Holland (1996) 313-385.

[30] P. Hauret and P. Le Tallec, Energy-controlling time integration methods for nonlinear elastodynamics and low-velocity impact. Comput. Methods Appl. Mech. Engrg. 195 (2006) 4890-4916.

[31] B. Heinrich and B. Jung, Nitsche mortaring for parabolic initial-boundary value problems. Electron. Trans. Numer. Anal. 32 (2008) 190-209.

[32] P. Heintz and P. Hansbo, Stabilized Lagrange multiplier methods for bilateral elastic contact with friction. Comput. Methods Appl. Mech. Eng. 195 (2006) 4323-4333.

[33] H.B. Khenous, Problèmes de contact unilatéral avec frottement de Coulomb en élastostatique et élastodynamique. Etude mathématique et résolution numérique. Ph.D. thesis, INSA de Toulouse (2005).

[34] H.B. Khenous, P. Laborde, and Y. Renard, Mass redistribution method for finite element contact problems in elastodynamics. Eur. J. Mech. A Solids 27 (2008) 918-932.

[35] N. Kikuchi and J.T. Oden, Contact problems in elasticity: a study of variational inequalities and finite element methods. In vol. 8 of SIAM Stud. Appl. Math. Society for Industrial and Applied Mathematics (SIAM), Philadelphia, PA (1988).

[36] J.U. Kim, A boundary thin obstacle problem for a wave equation. Comm. Partial Differ. Equ. 14 (1989) 1011-1026.

[37] T.A. Laursen, Computational contact and impact mechanics. Springer-Verlag, Berlin (2002).

[38] T.A. Laursen and V. Chawla, Design of energy conserving algorithms for frictionless dynamic contact problems. Int. J. Numer. Methods Eng. 40 (1997) 863-886.

[39] G. Lebeau and M. Schatzman, A wave problem in a half-space with a unilateral constraint at the boundary. J. Differ. Equ. 53 (1984) 309-361.

[40] J. Nitsche, Über ein Variationsprinzip zur Lösung von Dirichlet-Problemen bei Verwendung von Teilräumen, die keinen Randbedingungen unterworfen sind. Abhandlungen aus dem Mathematischen Seminar der Universität Hamburg 36 (1971) $9-15$.

[41] C. Pozzolini, Y. Renard, and M. Salaün, Vibro-impact of a plate on rigid obstacles: existence theorem, convergence of a scheme and numerical simulations. IMA J. Numer. Anal. 33 (2013) 261-294.

[42] Y. Renard, The singular dynamic method for constrained second order hyperbolic equations: application to dynamic contact problems. J. Comput. Appl. Math. 234 (2010) 906-923. 
[43] Y. Renard, Generalized Newton's methods for the approximation and resolution of frictional contact problems in elasticity. Comput. Meth. Appl. Mech. Engrg. 256 (2013) 38-55.

[44] R. Stenberg, On some techniques for approximating boundary conditions in the finite element method. In proc. of International Symposium on Mathematical Modelling and Computational Methods Modelling 94 (Prague, 1994). J. Comput. Appl. Math. 63 (1995) 139-148.

[45] V. Thomée, Galerkin finite element methods for parabolic problems. In vol. 25 of Springer Ser. Comput. Math. Springer-Verlag, Berlin (1997).

[46] B. Wohlmuth, Variationally consistent discretization schemes and numerical algorithms for contact problems. Acta Numer. (2011) 569-734.

[47] P. Wriggers, Computational Contact Mechanics. Wiley (2002).

[48] P. Wriggers and G. Zavarise, A formulation for frictionless contact problems using a weak form introduced by Nitsche. Comput. Mech. 41 (2008) 407-420. 